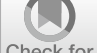

Check for

updates

Cite as

Nano-Micro Lett.

(2019) 11:13

Received: 13 December 2018

Accepted: 28 January 2019

Published online: 18 February 2019

(C) The Author(s) 2019

\title{
Recent Progress in the Fabrication, Properties, and Devices of Heterostructures Based on 2D Materials
}

\author{
Yanping Liu ${ }^{1}$, Siyu Zhang ${ }^{1}$, Jun He ${ }^{1}$, Zhiming M. Wang ${ }^{2}$, Zongwen Liu $^{3} \bowtie$ \\ Yanping Liu and Siyu Zhang have contributed equally to this work. \\ $\triangle$ Yanping Liu, liuyanping@csu.edu.cn; Zhiming M. Wang, zhmwang@gmail.com; Zongwen Liu, \\ zongwen.liu@sydney.edu.au \\ 1 School of Physics and Electronics, Hunan Key Laboratory for Super-Microstructure and Ultrafast Process, \\ Central South University, 932 South Lushan Road, Changsha 410083, Hunan, People's Republic of China \\ 2 State Key Laboratory of Electronic Thin Films and Integrated Devices, University of Electronic Science \\ and Technology of China, Chengdu 610054, People's Republic of China \\ 3 School of Chemical and Biomolecular Engineering, The University of Sydney, Sydney, NSW 2006, \\ Australia
}

\section{HIGHLIGHTS}

- The controllable fabrication methods, the unique properties, and relative applications of 2D heterostructures were summarized.

- The generation and detection of interlayer excitons in 2D heterostructures with type II band alignment indicate a longer lifetime and larger binding energy than intralayer excitons.

- The advances in magnetic tunneling junctions based on 2D heterostructures can be applied in spintronic devices to realize spin filtering.

\begin{abstract}
With a large number of researches being conducted on two-dimensional (2D) materials, their unique properties in optics, electrics, mechanics, and magnetics have attracted increasing attention. Accordingly, the idea of combining distinct functional 2D materials into heterostructures naturally emerged that provides unprecedented platforms for exploring new physics that are not accessible in a single $2 \mathrm{D}$ material or $3 \mathrm{D}$ heterostructures. Along with the rapid development of controllable, scalable, and programmed synthesis techniques of high-quality 2D heterostructures, various heterostructure devices with extraordinary performance have been designed and fabricated, including tunneling transistors, photodetectors, and spintronic devices. In this review, we present a summary of the latest progresses in fabrications, properties, and applications of different types of 2D heterostructures, followed by the discussions on present challenges and perspectives of further investigations.
\end{abstract}

KEYWORDS Two-dimensional (2D) materials; 2D heterostructures; Charge and magnetotransport; Electronic and optoelectronic devices

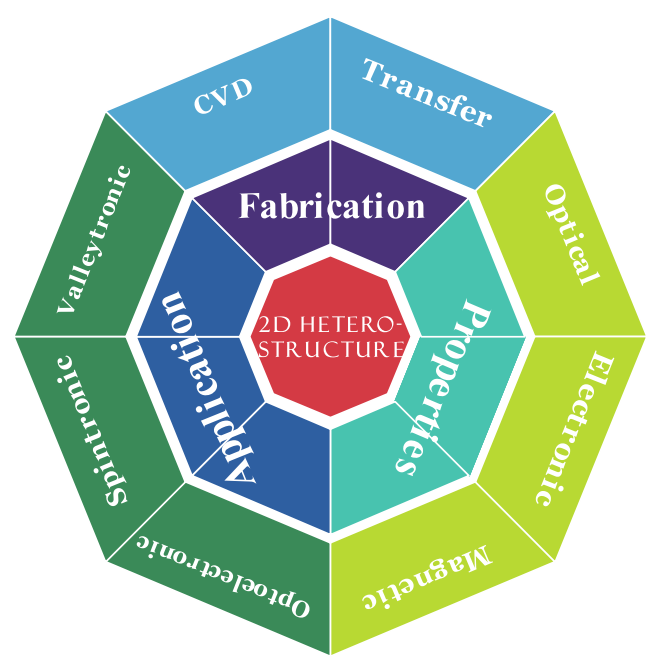




\section{Introduction}

Since the successful preparation of graphene in 2004 [1], the properties and applications of two-dimensional (2D) materials have attracted much attention. 2D materials can be divided into single-element 2D materials (such as graphene, black phosphorus (BP), silylene, germanene, etc.) and compound 2D materials (TMDs, hBN, TMCs, III-V group elements, compound semiconductor, etc.) [2]. Compared to bulk materials, layered 2D materials possess many peculiar properties that are strongly related to their number of layers. For example, the band structure at the Dirac point of a monolayer of graphene exhibits a linear dispersion relation, which is quite different from the parabolic band structure of double-layered grapheme [3]. And for black phosphorus (BP), the bandgap displays an evident redshift as the number of layers increases [4]. Besides, the direct-to-indirect band gap transition has been demonstrated in TMD semiconductors when it changes from single layer to multilayer [5]. 2D thin-layered materials have been considered as promising building blocks for the next generation of electronic and optoelectronics devices due to their extraordinary properties. In particular, 2D atomically thin structures are immune from the short channel effect and their mechanical strength allows for integration into flexible and wearable circuits $[6,7]$.

However, a number of issues limit the application of single 2D materials. Specifically, the direct deposition of metal electrodes on 2D semiconductors during device fabrication results in a high contact resistance due to the Schottky barrier [8-10]. Also, the intralayer excitons generated in single 2D semiconductor materials are hard to manipulate due to their short lifetime [11], which restricts their applications in exciton devices. Furthermore, hBN and most insulated 2D materials are not suitable to be applied in devices alone, and BP is easily oxidized when exposed to air.

Therefore, the idea of that 2D materials can be assembling 2D materials into heterostructures was put forward [12], and many novel properties of these heterostructures have been discovered [13-19]. Heterostructured 2D materials can be divided into two categories: the vertically stacked heterostructures and the epitaxial grown planar heterostructures. In this paper, we focus on the fabrication methods, the properties, and the applications of $2 \mathrm{D}$ heterostructures in these two types.

\section{Fabrications of 2D Heterostructures}

\subsection{Deterministic Transfer Method}

The layered 2D materials prepared through mechanical exfoliation and chemical vapor deposition can be transferred onto different substrates at a desired location. This usually requires a long working distance optical inspection system in combination with an XYZO direction micromanipulator for accurate placement and certain polymer layers as a transfer medium. There are four types of polymer carriers: (I) PMMA/sacrifice layer [12, 20], (II) PDMS [21, 22], (III) thermoplastic polymer [23], and (IV) hybrid stamp composed of PDMS/PPC (or PC, PMMA)/ hBN [24]. The transfer processes are slightly different from each other as schematically illustrated in Fig. 1.

Step 1: The polymer carriers are spin-coated layer by layer onto a $\mathrm{Si} / \mathrm{SiO}_{2}$ substrate (mainly for type I transfer media) or a glass slide and then mechanically exfoliate thin flakes of 2D nanostructures onto the carriers; Step 2: mount the glass slide on the micro-manipulator directly or after the wet transfer process (mainly for type I carriers, the PMMA with flakes is attached to a glass slide when released from the $\mathrm{Si} / \mathrm{SiO}_{2}$ substrate through desolating the sacrificial layer) and then align the flakes on the polymer carriers with the target material prepared on existing substrate into the desired location and orientation under microscope with micro-manipulate arms, and then the glass slide was lowered down until the two flakes make contact to form the van der Waals heterostructure; Step 3: directly pick up the target flakes and combine with another layered material to form multi-heterostructure (for type IV carriers) or release the heterostructure by slowly lifting the glass slide and remove the residual polymer in acetone solution. (For type III and type IV transfer media, heating substrates are needed to release the stacking.)

The comparison between the different deterministic transfer methods is presented in Table 1 [25]. The cleanness is mainly determined by whether the interfaces in the heterostructure are exposed to the polymer. And the easiness and speed are dependent on the number of steps and specific procedures such as spin coating, heating, and wet transferring, which will increase the complexity and slow down the speed to some extent. The PDMS dry transfer is considered the easiest and 

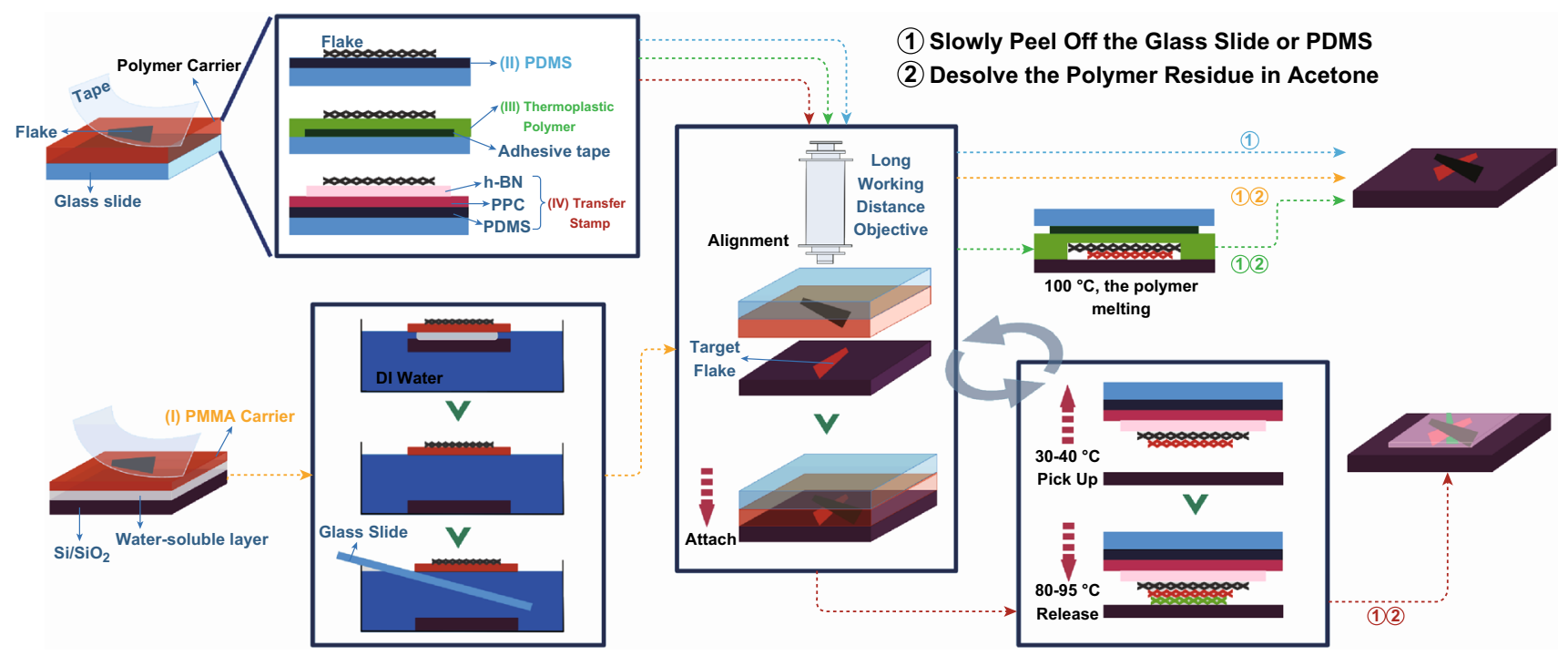

Fig. 1 The deterministic transfer method. The yellow, blue, green, and red dashed lines correspond to the process of transfer using polymer carriers of types I to IV, respectively, and in the end a bare heterostructure or a multilayer heterostructure covered by hBN will be fabricated. (Color figure online)

Table 1 Comparison between the different deterministic placement methods ${ }^{1}$. Reprinted with permission from Ref. [25]

\begin{tabular}{|c|c|c|c|}
\hline Carrier type & Cleanness & Easiness & Speed \\
\hline PMMA/sacrifice layer & $\boldsymbol{\Delta} \boldsymbol{\Delta} \boldsymbol{\Delta}$ & $\boldsymbol{\Delta} \boldsymbol{\Delta} \boldsymbol{\Delta}$ & $\boldsymbol{\Delta} \boldsymbol{\Delta} \boldsymbol{\Delta}$ \\
\hline PDMS & $\boldsymbol{\Delta} \boldsymbol{\Delta} \boldsymbol{\Delta}$ & $\boldsymbol{\Delta} \boldsymbol{\Delta} \boldsymbol{\Delta} \boldsymbol{\Delta} \boldsymbol{\Delta}$ & $\boldsymbol{\Delta} \boldsymbol{\Delta} \boldsymbol{\Delta} \boldsymbol{\Delta} \boldsymbol{\Delta}$ \\
\hline Thermoplastic polymer & $\Delta$ & $\Delta \boldsymbol{\Delta}$ & $\Delta \boldsymbol{\Delta} \boldsymbol{\Delta}$ \\
\hline $\begin{array}{l}\text { PDMS/PPC (or PC, } \\
\text { PMMA)/hBN }\end{array}$ & $\boldsymbol{\Delta} \boldsymbol{\Delta} \boldsymbol{\Delta} \boldsymbol{\Delta} \boldsymbol{\Delta}$ & $\boldsymbol{\Delta}$ & $\Delta \boldsymbol{\Delta}$ \\
\hline
\end{tabular}

${ }^{1}$ The numbers of symbol $\boldsymbol{\Delta}$ indicate the degrees in cleanness, easiness and speed

quickest method, as the progress does not involve spin coating, wet transfer, or heating process. PDMS films have become commercialized commodities, and their viscosity is strongly related to the speed when being peeled off [26]. Meanwhile, the pickup method remains the best way to assemble multi-heterostructure with no residual polymer at interfaces. In conclusion, the deterministic transfer method has high flexibility in fabricating various heterostructures, but its drawbacks are obvious. It is inevitable to cause polymer residue at the interface or on the surface, and the 2D material samples may be destroyed or got wrinkled during the transfer process. Another challenge of the deterministic transfer method is the control of the stack orientation.

\subsection{Chemical Vapor Deposit (CVD) Growth}

CVD synthesis is a bottom-up strategy for the preparation of vertical 2D heterostructures and is able to realize the growth of planar multi-junction heterostructures. In order to obtain an atomically sharp heterojunction and a clean interface in vertically stacked heterostructures, as well as achieve scalable and controllable fabrication of both vertical and planar heterostructures, various approaches have been explored to realize CVD growth of heterostructures.

\subsubsection{The One-Step CVD Method}

The schematic diagram of Fig. 2a shows the processes of this method, which is suitable for preparing heterostructures with the component materials containing the same elements. Gong et al. [27] successfully fabricated both vertical and planar $\mathrm{MoS}_{2} / \mathrm{WS}_{2}$ heterostructures by this one-step CVD method that demonstrated the in-plane epitaxial growth at a lower temperature of $650{ }^{\circ} \mathrm{C}$, as the provided energy was not enough for the nucleation of $\mathrm{WS}_{2}$ on the surface of $\mathrm{MoS}_{2}$, but at a higher temperature of $850^{\circ} \mathrm{C}$ vertically stacked structure could be formed as the van der Waals heterostructure was more thermodynamically stable. 
(a)

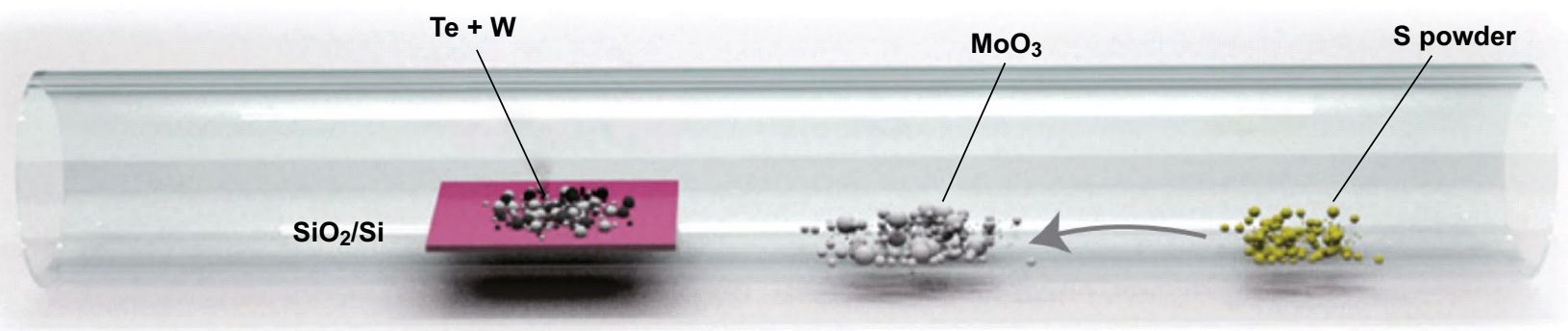

Quartz tube

(b)

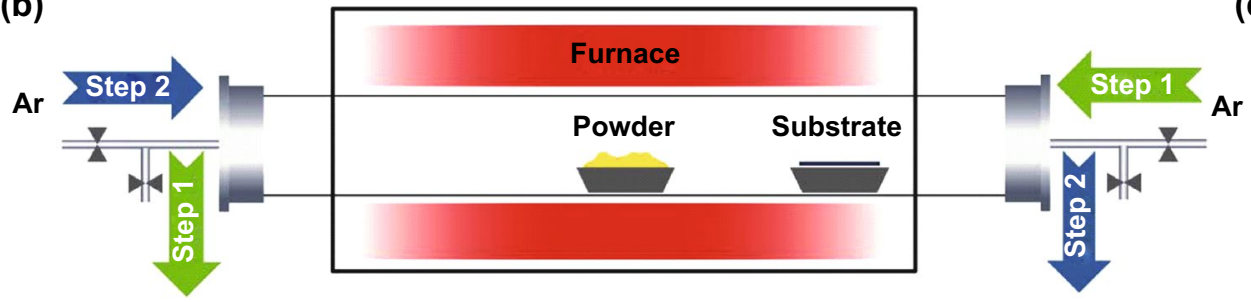

(c)

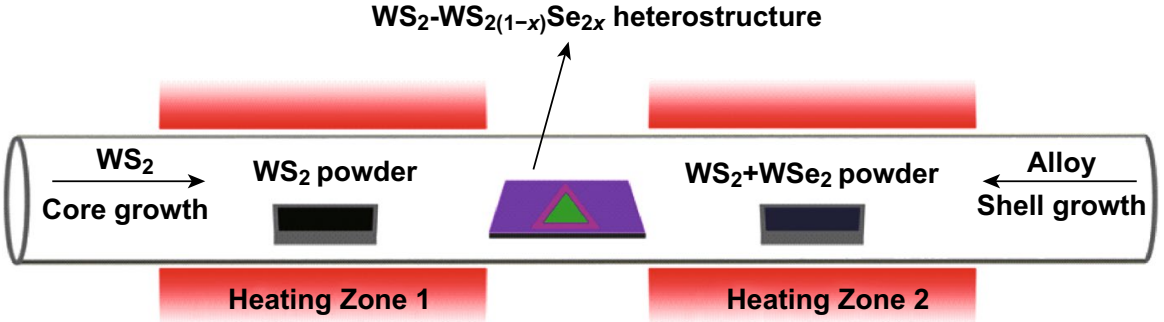

(e)

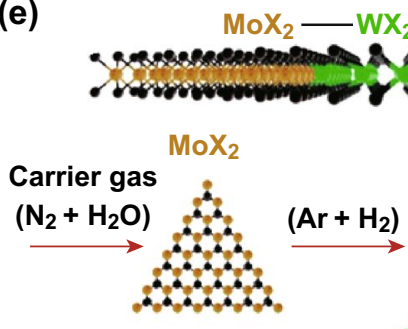

(d)

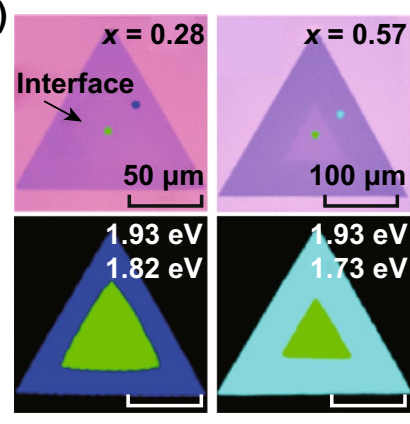

(f)

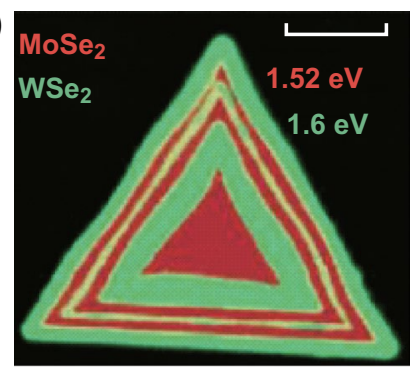

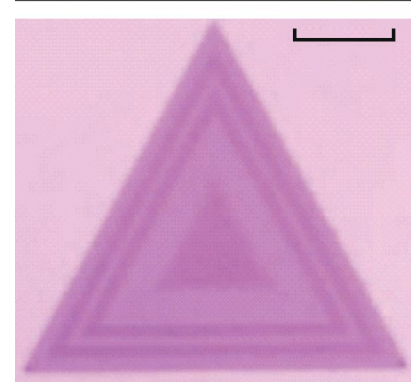

Fig. 2 Chemical vapor deposit (CVD) growth. a The diagram of the synthesis of $\mathrm{MoS}_{2} / \mathrm{WS}_{2}$ heterostructures through one-step CVD method. $\mathrm{S}$ powder is placed at the upstream, and a wafer with mixed powder of $\mathrm{W}$ and Te is put downstream. Reprinted with permission from Ref. [27]. b Multi-step CVD growth of realized through direction-switchable carrier flow and cooling process. Reprinted with permission from Ref. [32]. c the modulable growth of $\mathrm{WS}_{2}-\mathrm{WS}_{2(1-x)} \mathrm{Se}_{2 x}(0<x \leq 1)$ monolayer lateral heterostructures using dual heating quartz tube and $\mathbf{d}$ the optical picture and PL intensity mappings of heterostructures with different $x$. Reprinted with permission from Ref. [30]. e The principle of one-pot synthesis strategy and $\mathbf{f}$ the optical picture and PL intensity mappings of the hetero-superlattice indicating the sharp interlines. Reprinted with permission from Ref. [31]

\subsubsection{The Two-Step CVD Method}

This is a more commonly adopted approach using the as-grown layered crystals as a substrate for the second layer. Such procedures were also adapted for both the vertically stacked and lateral heterostructures that are mainly affected by the rate of gas flow and the synthesis time. Li et al. [28] reported the growth of 2D GaSe/MoSe heterostructure by this two-step CVD method. Such MX/ $\mathrm{MX}_{2}$ vertical heterostructures exhibit incommensurate 
superstructures because of the large lattice misfit between the two layers. In addition, the synthesis of a stacked TMD/hBN heterostructure was also realized by using the $\mathrm{Ni}-\mathrm{Ga}$ alloy and Mo foil as the substrate without any intermediate operations. In such a case, the Ni-Ga alloy promoted the formation of the $\mathrm{hBN}$ honeycomb lattice, while the Mo foil was a source of Mo [29].

\subsubsection{The Multi-step CVD Method}

By switching the direction and modulating the components of gas flow [30-32], the multi-step CVD method was developed (Fig. 2b). This approach makes the boundary of heterojunctions sharper and enables the sequential growth of the multi-junction heterostructure. Biyuan Zheng et al. [30] reported an efficient method to grow modulable $\mathrm{WS}_{2}-\mathrm{WS}_{2(1-x)} \mathrm{Se}_{2 x}(0<x \leq 1)$ monolayer planar heterostructure (Fig. 2c) with tunable band alignment (Fig. 2d). Using the dual heating furnace, the switching of the synthesis from a pure $\mathrm{WS}_{2}$ growth to a $\mathrm{WS}_{2(1-x)} \mathrm{Se}_{2 x}$ $(0<x \leq 1)$ alloy formation can be controlled by changing the direction and the temperature of the Ar gas flow. The parameter $x$ is modulated by the ratio of the mixed $\mathrm{WS}_{2} /$ $\mathrm{WSe}_{2}$ powders.

Moreover, the control of growth can also be realized by changing the components of the gas flow. Sahoo et al. [31] reported the one-pot synthesis strategy by placing two precursors ( $\mathrm{MoX}_{2}$ and $\mathrm{WX}_{2}$ mixed powders) in the same boat at the heating zone, while the substrate was held at a lower temperature. The mechanism is vividly illustrated in Fig. 2e: under the $\mathrm{N}_{2}+\mathrm{H}_{2} \mathrm{O}(\mathrm{g})$ gas flow, only the growth of $\mathrm{MoX}_{2}$ was promoted. When the gas flow was switched to the $\mathrm{Ar}+\mathrm{H}_{2}(5 \%)$ gas flow, only the growth of $\mathrm{WX}_{2}$ was allowed. Reversing the gas flow would terminate the current reaction. Figure $2 \mathrm{f}$ shows the optical images and the Raman intensity maps at 240 and $250 \mathrm{~cm}^{-1}$ revealing the well-defined spatial distribution and sharp interface of the $\mathrm{MoSe}_{2}$ and $\mathrm{WSe}_{2}$ domains in the multi-junction heterostructure. Compared with the one-step and the two-step CVD methods, the multi-step CVD method is more flexible and controllable that enables the possibility of creating spatially selected optoelectronic devices due to the separation of electrons and holes into different materials.

\section{Properties}

\subsection{Band Alignment}

The band alignment is the fundamental property of 2D heterostructures. Most charge transport behavior and illuminance properties are originated from the band structures, especially the band alignment. The band energy can be calculated in theory based on the first principles and measured through $\mu$-XPS. Figure $3 a$ [33] shows the band alignments of a monolayer semiconducting TMDs and monolayer $\mathrm{SnS}_{2}$ calculated by Perdew-Burke-Ernzerhof (PBE) with spin-orbit coupling (SOC). The results indicate that, for the monolayer TMDs in $\mathrm{MX}_{2}$ form, the energy of the conduction band minimum (CBM) and the valence band maximum (VBM) increases with the atomic number of $\mathrm{X}$. Thus, when two different materials with sizable band gaps get combined into a heterostructure, there will be three types of I-III band alignments without considering the band bending at the interface, which are called straddling gap, staggered gap, and broken gap, respectively. The type I band alignment contributes to the fast recombination of electrons and holes that allows them to be used in luminescent devices, such as light-emitting diodes (LEDs) [34, 35]. The type II band alignment can facilitate the effective spatial separation of electrons and holes, prolonging the lifetime of interlayer excitons and making them a good candidate for the application in electron-hole separators and related optoelectronic devices. The type III band alignment allows the band-to-band tunneling (BTBT) effect of carriers and enables the operation of the tunnel field effect transistors (TFET). Compared with the type II band alignment, the speed of the transportation of the electrons and holes in type III heterostructures is much faster that results in a large number of electrons and holes separating into different layers of the material, so the heterojunction displays semi-metallic features generating a strong built-in electric field that makes the type III heterostructures ideal for new-generation thermal photovoltaic cells.

Interestingly, when taking the band bending at the interface into consideration, the band structure of the heterostructures presents more novel properties. For example, when two semiconductors form a PN junction with type I band alignment (Fig. 3b), the VB and the CB on the different sides will bend in the opposite directions, forming a 
(a)

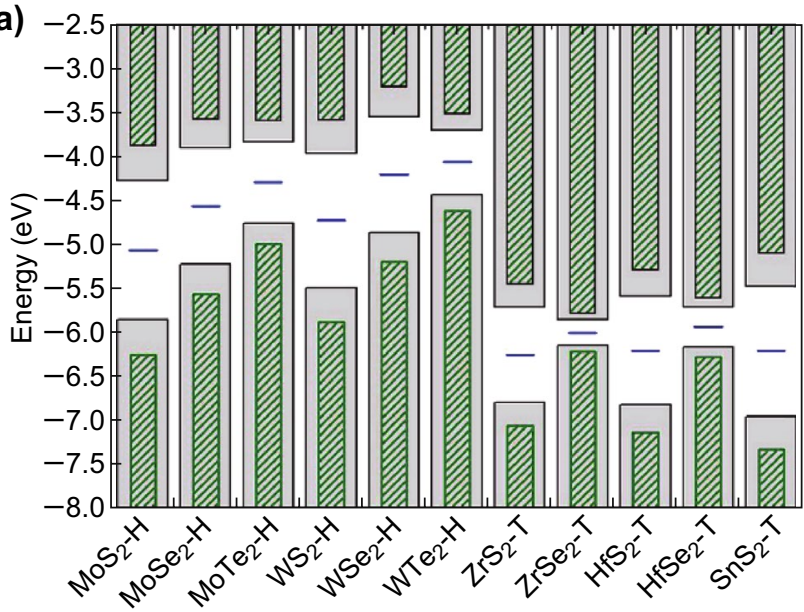

(c)

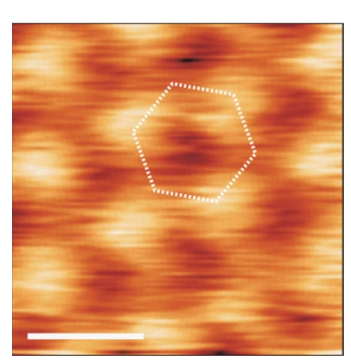

(e)

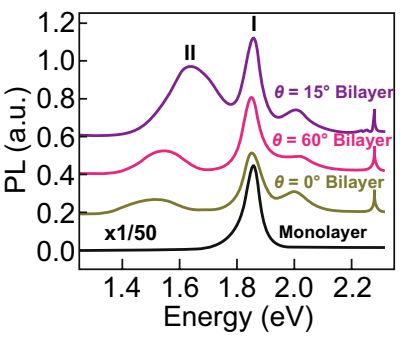

(d)

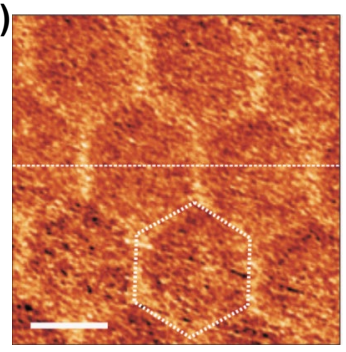

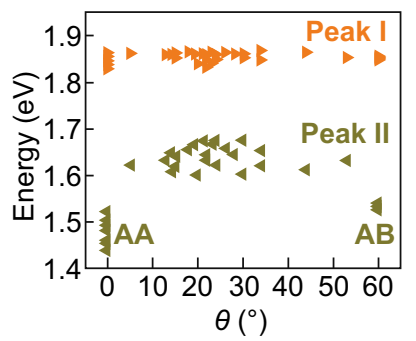

(f)

(b)
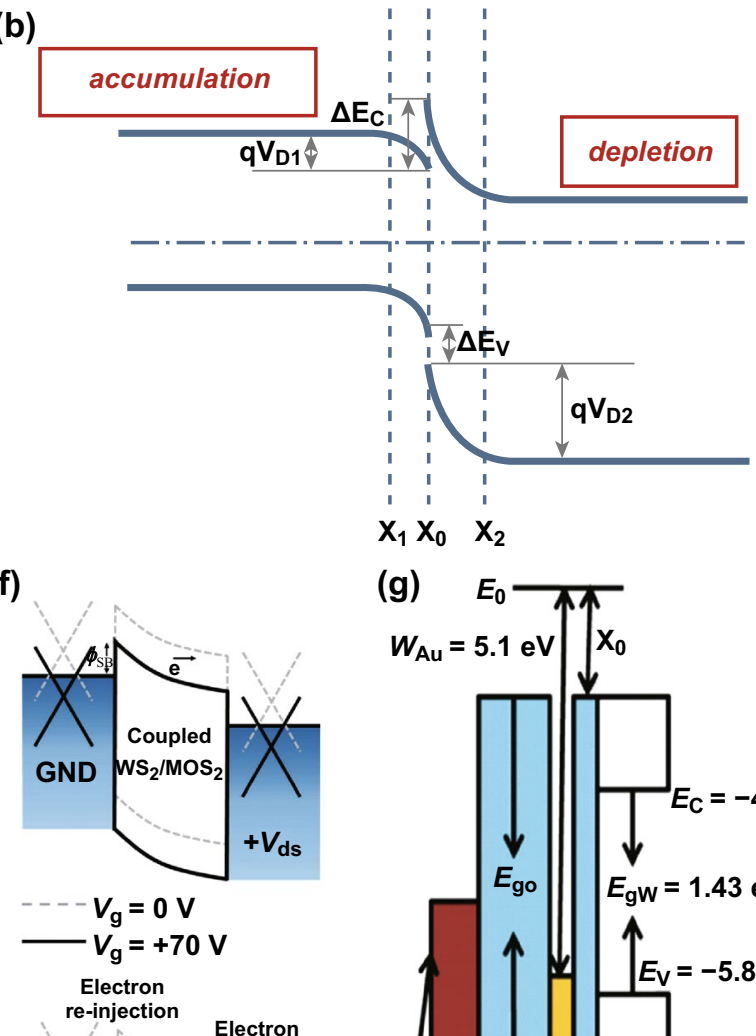

(g) $E_{0} \longrightarrow$ 不

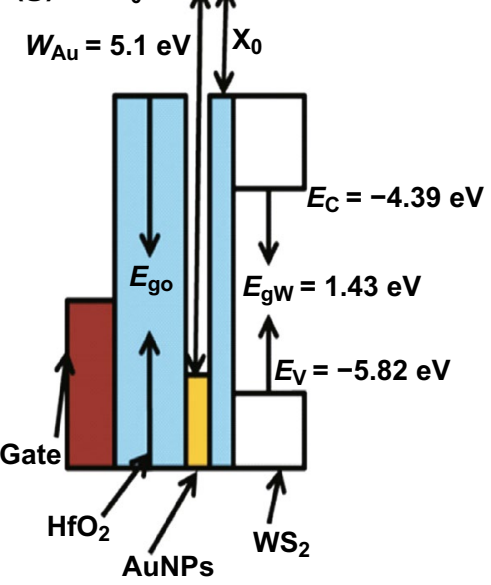

Fig. 3 Band alignment and band engineering methods. a Theoretical positions of VBM and CBM of VIB- and IVB-TMDs calculated by PBESOC. Reprinted with permission from Ref. [33]. b Schematic diagram of band bending at the interface, forming a notch on the accumulation side and a peak on the depletion side. c, d STM measurements of hBN/graphene heterostructure stacked with the different rotation angle of $5^{\circ}$ (c) and $0^{\circ}(\mathbf{d})$. Reprinted with permission from Ref. [41]. e PL spectra of $\mathrm{MoS}_{2}$ monolayer and bilayers with different twist angles, where peak I is related to the direct transition at $K_{-}$valley and peak II may be associated with the indirect band gap. Reprinted with permission from Ref. [41]. f The principle of photogating band alignment engineering. Reprinted with permission from Ref. [42]. $\mathbf{g}$ The band structure of a floatinggate device based on monolayer $\mathrm{WS}_{2}$. Reprinted with permission from Ref. [43]

notch and a peak, respectively. There is none symmetry in $\Delta E_{\mathrm{C}}$ and $\Delta E_{\mathrm{V}}$ values which will cause different potential barriers for electrons and holes.

In addition, the band structure of a $2 \mathrm{D}$ heterostructure can be affected by many factors. Firstly, the quality of the interface is very important. For example, the interface defects and impurities can cause the defect or impurity energy level; and intrinsic metal-induced gap states (MIGS) or extrinsic disorder induced gap states (DIGS) at the interface would lead to the occurrence of Fermi-level pinning effect [36]. Bampoulis et al. [37] demonstrated that the subsurface metal-like defects in $\mathrm{MoS}_{2} /$ metal heterostructures could hugely decrease the Schottky barrier height (SBH) that attributed to strong Fermi-level pinning at the defects. On the other hand, a weak Fermi-level pinning led to the SBH modulation by electrical gating [38], and a very weak Fermi-level pinning has been observed at the graphene/TMD interfaces. A shift of about $120 \mathrm{mV}$ 
was observed between the spectra of scanning tunneling spectroscopy (STS) of flakes residing on single-layer graphene (SLG) and bilayer graphene (BLG) substrates, which is equal to the difference in the work function of SLG and BLG. Secondly, the lattice constants of the materials and their stake orientation are also important factors. When two 2D materials with similar lattice constants form heterostructures, such as graphene/hBN [39] and $\mathrm{SnS}_{2}$ / $\mathrm{MoS}_{2}$ [40], a periodic Moiré pattern will be formed. Figure $3 \mathrm{c}, \mathrm{d}$ shows the STM measurements on graphene/hBN heterostructure with a rotation smaller (Fig. 3d) and larger (Fig. 3c) than $1^{\circ}$. The later one displays a nearly same spatial periodic behavior exhibiting a much larger Moiré pattern due to the high Young modulus of graphene. Such deformation of lattice structure results in a much stronger van der Waals interaction and causes extra periodic potential in graphene that adjusts its band structure. Moreover, the indirect band gap varies appreciably with the stacking orientation [41]: the largest redshift for AA-(twist angle of $0^{\circ}$ ) and $\mathrm{AB}$-(twist angle of $60^{\circ}$ ) stacked bilayers and a significantly smaller but constant redshift for all other twist angles (Fig. 3e).

However, adjusting the band alignment through the above structural factors is complex and inflexible. The band structure of heterojunction can also be externally controlled by an electric field, magnetic field and light field [44]. The band engineering regulated by the gate voltage leads to the transitions in 2D heterostructures between band alignments of type I-III. Particularly, graphene has a unique linear dispersion relationship with a finite DOS; thus, the Fermi level and work function of graphene are gate modulable, making graphene widely used as the contact between the electrodes and the channel of the 2D material to reduce the contact resistance $\left(R_{\mathrm{c}}\right)$. What's more, band structure can also be optically modulated to behave like a photogate, a phenomenon looking like the addition of a local gate $\left(\Delta V_{\mathrm{g}}\right)$ to the device when under illumination [45]. The band diagram of a photodetector with the $\mathrm{WS}_{2} / \mathrm{MoS}_{2}$ hetero-bilayer as a light absorption layer and the graphene as the electrode contact is shown in Fig. 3f [42]. Under illumination, the accumulation of holes at the region acts as a positive gate on the source graphene electrode, effectively raising the Fermi level of the graphene electrode and lowering the SBH. Figure 3g [43] shows a typical band structure of floating-gate devices with an Au floating-gate layer to form energy well to trap the charges.

\subsection{Charge Transport Properties in 2D Heterostructures}

Different band alignments result in a variety of charge transport characteristics accompanied by the process of energy transfer. On the one hand, the single-particle transportation, including interlayer tunneling effect and charge trapping phenomena, has been widely reported and novel devices based on those transportation mechanisms have been fabricated that exhibit low energy-assumption with high performance; on the other hand, the many-body transport and the separation of electron-hole pairs in 2D heterostructures have also been theoretically predicted and practically demonstrated [11], which enable the prospect of broad applications in optoelectronic field [46-50].

\subsubsection{Single-Particle Transports}

The ultra-narrow channel length and atomically sharp interfaces can realize band-to-band tunneling (BTBT) effect by electrostatic gating to avoid deprivation of band-edge sharpness resulted from chemical doping [51]. As shown in Fig. 4a-c [52], when applying a small $V_{\mathrm{g}}$ (Fig. 4b), the CBM of $\mathrm{WSe}_{2}$ is higher than the VBM of $\mathrm{SnSe}_{2}$. The electrons in $\mathrm{SnSe}_{2}$ cannot tunnel into $\mathrm{WSe}_{2}$, but diffusion of charges occurs at the interface, corresponding to channel current and high resistance state, respectively, when applied with a positive and negative bias voltage. This corresponds to the off state of the device. When keeping on increasing the $V_{\mathrm{g}}$ while the CBM of $\mathrm{WSe}_{2}$ is set below the VBM of $\mathrm{SnSe}_{2}$ (Fig. 4c), a tunneling window is opened, such that an interlayer tunneling can flow from $\mathrm{SnSe}_{2}$ to $\mathrm{WSe}_{2}$. In type I band alignment, the layer with a wide bandgap will cause spatial confinement of electrons and holes in the "well" layer with a narrow bandgap [53] that causes a strong PL in a trapping layer and a quenched PL in the other (Fig. 4d).

When taking the band bending into consideration, the interfacial charge trapping can be seen in the PN junction of type II band alignment. Cai et al. [54] developed an electricgating switchable photodetector based on $\mathrm{p}-\mathrm{MSB} / \mathrm{WSe}_{2}$ by epitaxial growth of 2D van der Waals, in which the p-MSB serves as a light absorber, while in the $\mathrm{p}-\mathrm{MSB} / \mathrm{WSe}_{2}$ heterostructure an interfacial energy barrier and a band bending 
(a)

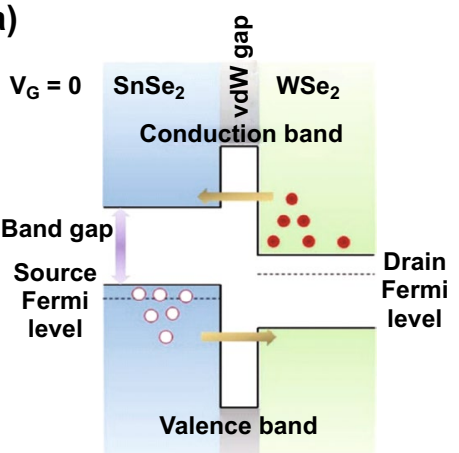

(b)

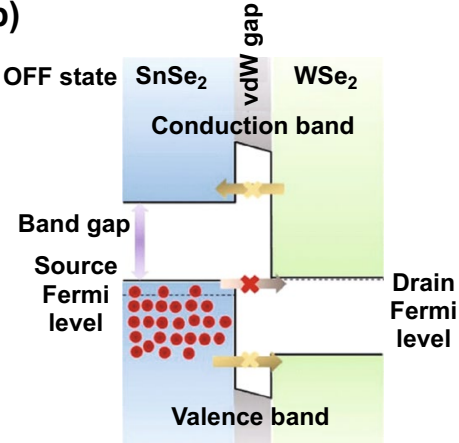

(e)

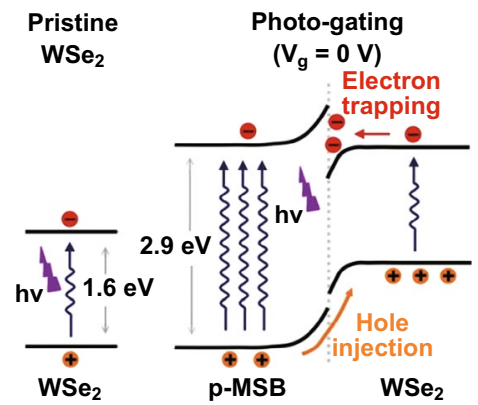

(c)

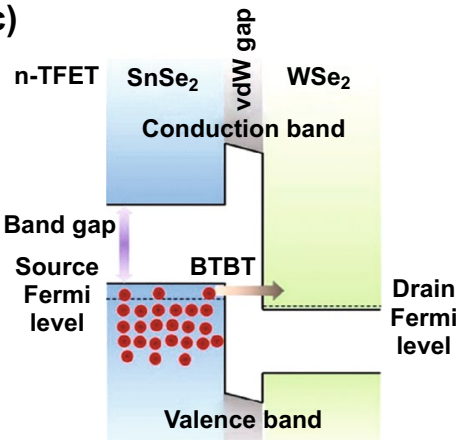

(f)

Large photoelectric-gating $\left(V_{g}=-60 \mathrm{~V}\right)$

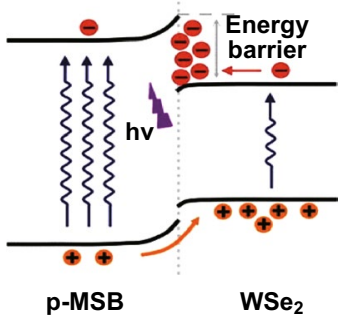

(g) GrBNS GrBN MoS 2 GrBND
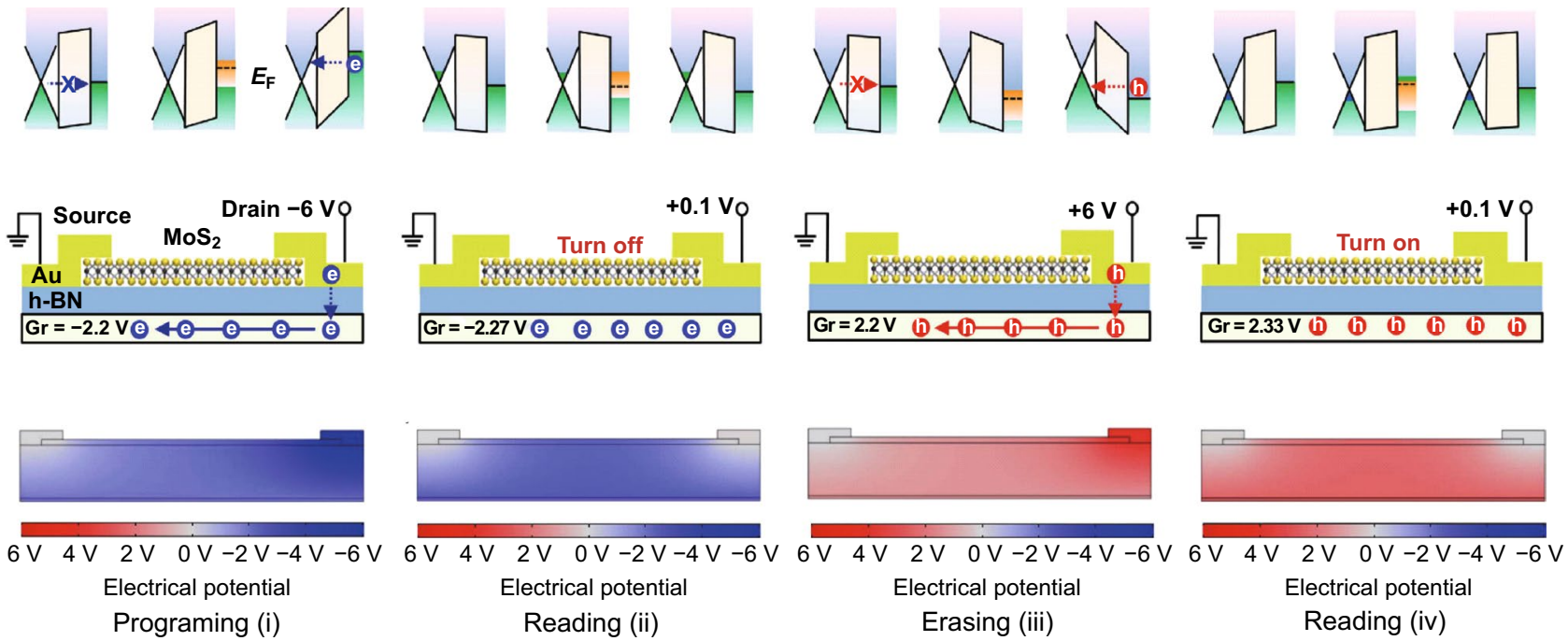

Fig. 4 The single-particle transport caused by different band structures. a-c The electronic modulation of BTBT in a TFET based on 2D SnSe $e_{2}$ $\mathrm{WSe}_{2}$ heterostructure. Reprinted with permission from Ref. [52]. d PL measurement of a stacked $\mathrm{WSe}_{2} / \mathrm{MoTe}_{2}$ heterostructure with proper annealing, where the blue, black, and red lines correspond to the PL intensity of specific energy (eV) from the isolated $\mathrm{WSe}_{2}$ region, isolated $\mathrm{MoTe}_{2}$ region, and hetero-stacked region, respectively. Reprinted with permission from Ref. [53]. e, $\mathbf{f}$ The diagram of the charge transferring in $\mathrm{p}-\mathrm{MSB} / \mathrm{WSe}_{2}$ heterostructure under illumination. Reprinted with permission from Ref. [54]. $\mathbf{g}$ The band alignment, charge transportation, and the distribution of the potential in the device. Reprinted with permission from Ref. [39]. (Color figure online)

between the lowest unoccupied molecular orbital of p-MSB and the $\mathrm{CBM}$ of $\mathrm{WSe}_{2}$ (Fig. 4e) enable unidirectional carrier injection. When a negative $V_{\mathrm{g}}$ is applied (Fig. 4f), the Fermi level of $\mathrm{WSe}_{2}$ shifts downward, leading to an increase of the interfacial energy barrier that enhances the interfacial charge trapping process. It is believed the interface quality is the key to avoid the on-state current decreasing under such plasma treatment. 
Another way to obtain charge trapping is to place a floating-gate layer, which is usually sandwiched with the tunneling and blocking dielectric layers, between the channel and control gate as a charge trapping layer [43, 55-60]. Figure 4g [61] shows the charge transport in the floating-gate structure with graphene as the trapping layer and monolayer $\mathrm{MoS}_{2}$ as the channel. When a $-6 \mathrm{~V}$ $(+6 \mathrm{~V})$ drain bias is applied, a large potential difference between the drain and graphene, and a nearly equal potential of the source electrode and graphene are generated. As a result, the electrons (and holes) are able to tunnel through the hBN layer at the drain electrode side and are trapped in the floating-gate layer, so are unable to tunnel back to the source electrode or the $\mathrm{MoS}_{2}$ channel.

\subsubsection{The Generation of Interlayer Excitons}

For the type II band alignment, the CBM and VBM are in different layers that lead to the spatial separation of electrons and holes. The ultrafast separation of electron-hole pairs could be detected by the pump-probe technique [48, $62,63]$. In the $\mathrm{WS}_{2} / \mathrm{MoS}_{2}$ heterostructure, after the $\mathrm{MoS}_{2}$ monolayer is directly pumped the photo-induced signal will appear instantaneously. The experimentally observed signal in the heterostructure shows a rising time that is shorter than 50 fs (Fig. 5a).

The detailed charge separation processes in the heterostructure are presented in Fig. 5c [62]. After the excitation of the $\mathrm{WS}_{2}\left(\mathrm{MoS}_{2}\right)$ monolayer, the electrons (holes) transferred from the CBM (VBM) of $\mathrm{WS}_{2}\left(\mathrm{MoS}_{2}\right)$ monolayer to the conduction (valence) bands just above (below) CBM (VBM) of the $\mathrm{MoS}_{2}\left(\mathrm{WS}_{2}\right)$ monolayer within $50 \mathrm{fs}$, generating a hot (a)
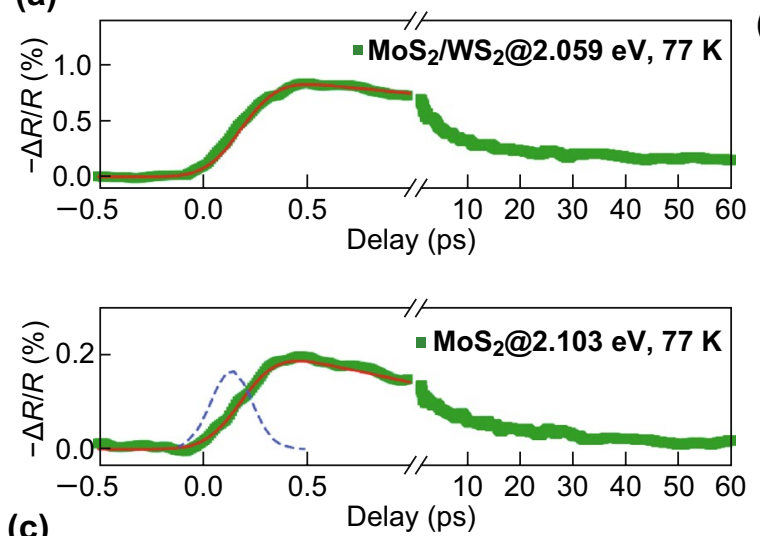

(c) (b)
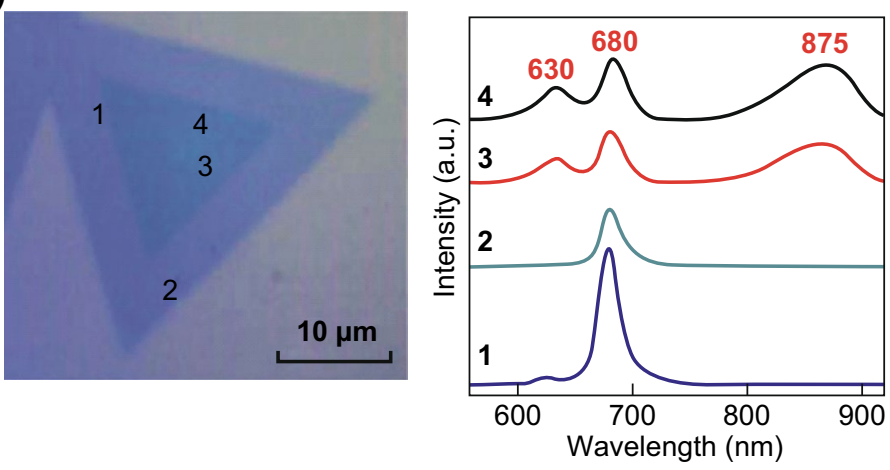

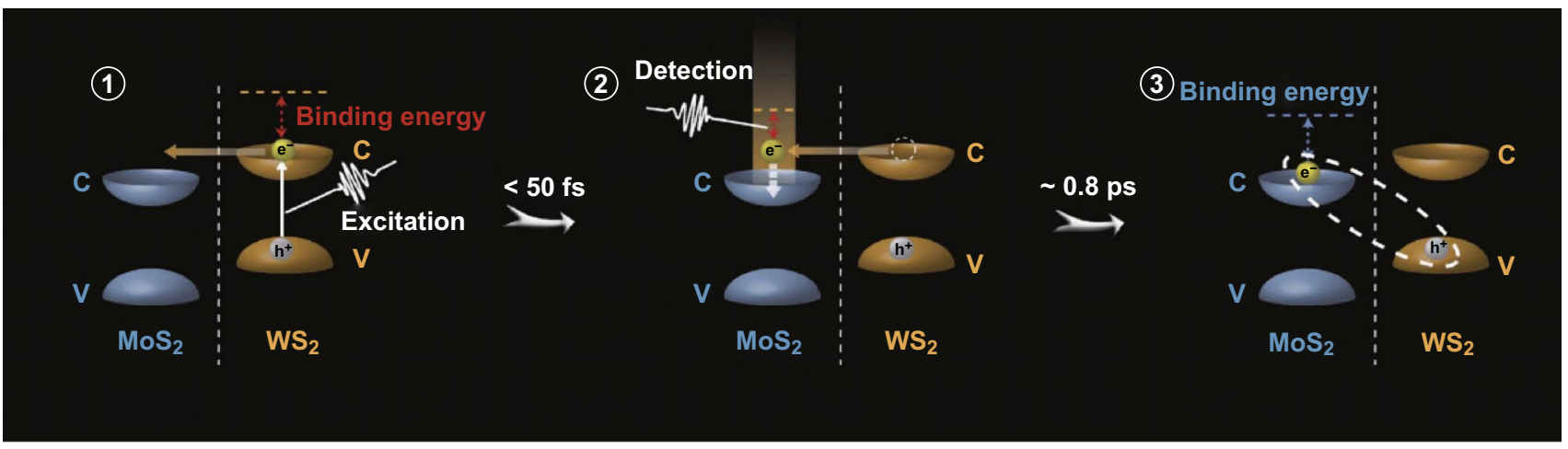

Fig. 5 The generation of interlayer excitons. a The transient absorption measurements of $\mathrm{WS}_{2} \mathrm{~A}$-exciton resonance in the MoS $/ \mathrm{WS}_{2}$ heterostructure and B-exciton resonance in an isolated $\mathrm{MoS}_{2}$ monolayer. The dynamic evolution signal is obtained by convoluting the instrument response function (blue dashed line). b PL spectra detected from the 1-4 region of a vertically stacked $\mathrm{WS}_{2} / \mathrm{MoS}_{2}$ heterostructure, and numbers 1 and 2 correspond to the $\mathrm{MoS}_{2}$ monolayer and number 3 and 4 regions are hetero-stacked bilayer. Two additional peaks at 630 and $875 \mathrm{~nm}$ indicate the generation of interlayer excitons. Reprinted with permission from Ref. [27]. $\mathbf{c}$ The two processes of the interlayer excitons generation. Reprinted with permission from Ref. [62]. (Color figure online) 
interlayer exciton with a relatively long distance. The hot excitons then reorganized and dissipated the excess energy to form the tightly bound interlayer excitons within $800 \mathrm{fs}$. The existence of the final tightly bound interlayer excitons could be distinctly demonstrated in photoluminescence spectra [62, 64] as shown in Fig. 5b [27]. The photoluminescence signal was taken from number 1 and 2 regions corresponding to the A-exciton resonances in the monolayer $\mathrm{MoS}_{2}$. But the signals from the heterostructure regions displayed exciton resonances in $\mathrm{MoS}_{2}$ and $\mathrm{WSe}_{2}$, leading to an additional interlayer exciton peak. The exciton photoluminescence signals of $\mathrm{MoS}_{2}$ and $\mathrm{WSe}_{2}$ were quenched in the heterostructure due to the charge transfer process.

\subsection{The Properties of Excitons in 2D Heterostructure}

The interlayer excitons hold the prolonged lifetime [50] and large binding energy which is strongly related to the distance between the two layers in vertical heterostructures. Simone Latini et al. [65] developed a QEH (quantum electrostatic heterostructure) model based on the $\mathrm{MoS}_{2} / x$-hBN/WSe vertical heterostructures. (The parameter $x$ is the number of hBN layers.) The binding energy of the interlayer excitons is calculated up to $0.3 \mathrm{eV}$ and decreases with the increasing parameter $x$, indicating a stable existence of the interlayer excitons at room temperature. But the weak oscillator strength and momentum-indirect nature make it challenging to directly observe the interlayer excitons by resonant optical excitation [66]. Figure 6a shows the spectra of electroluminescence (red line) and photoluminescence (black line) for lateral $\mathrm{p}-\mathrm{n}$ junctions based on a vertical $\mathrm{MoSe}_{2}-\mathrm{WSe}_{2}$ hetero-bilayer. The inset picture illustrates the electrode contacts of each layer. When applying a forward $V_{\text {sd }}$, the carriers would be injected in and recombine at the edge of the heterobilayer (orange arrows). It turned out that the photocurrent amplitude from the interlayer exciton was about 200 times smaller than that of the resonant excitation of the intralayer exciton.

Interlayer excitons also have ideal valley-contrast physics. Figure $6 \mathrm{~b}$ [17] shows the schematic of the interlayer exciton recombination in the $K_{ \pm}$valley in a $\mathrm{MoS}_{2} / \mathrm{WSe}_{2}$ heterostructure with the twist angle of $0^{\circ}$ and $60^{\circ}$. After circularly polarized light excites intralayer excitons in the $K_{+}$valleys of $\mathrm{MoS}_{2}$ and $K_{+}$valleys of $\mathrm{WSe}_{2}$, a fast interlayer charge hopping forms the interlayer exciton in the $K_{+}$ valley. The solid (dashed) arrows denote the dipole transition (interlayer hopping). Rivera et al. [67] observed long valley lifetime and valley drift-diffusion of host interlayer excitons in $\mathrm{MoSe}_{2}-\mathrm{WSe}_{2}$ heterostructures with small twist angles (Fig. 6d). The valley polarization is greatest at $+60 \mathrm{~V}$ and highly suppressed at $-60 \mathrm{~V}$. The valley polarization lifetime increases with $V_{\mathrm{g}}$, reaching $39 \pm 2 \mathrm{~ns}$ at $+60 \mathrm{~V}$, as determined by fitting a single exponential decay. The extraordinary valley-contract properties allow the possibility of the excitonic optoelectronic circuit to switch the valley functionalities and provide a platform for investigating excitonic superfluidity and condensation.

In addition to intralayer and interlayer excitons, there is the theoretical existence of trions in 2D heterostructures. Figure 6e shows the different kinds of combinations of trions in a $\mathrm{MoS}_{2} / \mathrm{WS}_{2}$ heterostructure. Thorsten Deilmann et al. [68] calculated the interlayer excitation in a $\mathrm{MoS}_{2} / \mathrm{WS}_{2}$ heterostructure and predicted the existence of bound interlayer trions below the neutral interlayer. The binding energies are 18 and $28 \mathrm{meV}$ for the positive and negative interlayer trions with both electrons/holes located on the same layer.

\subsection{Magnetic Properties in 2D Heterostructures}

For the low-dimensional magnetic materials, the coercivity, saturation magnetization, Curie temperature $\left(T_{\mathrm{C}}\right)$, and other magnetic parameters of the materials are related to the number of layers and grain size. Therefore, 2D materials usually present magnetic properties that differ from its bulk form. The application of 2D magnetic materials in heterostructures is of great significance for the study of spintronics, valleytronics, and electromagnetics [69]. The magnetic properties of 2D heterostructures are significant for the external ways to control (gate) the propagation of spin and valley (polarized) currents at room temperature. The massive theoretical calculations and experimental results indicate that by assembling $2 \mathrm{D}$ materials (graphene or TMDs) with ferromagnetic materials into a van der Waals heterostructure [70], a large magnetic exchange field can be generated at the interface and thus the regulation of the spin and valley pseudospin in 2D materials can be realized [71-73]. The existence of the magnetic exchange field can amplify the effect of the external magnetic field, which originates from the proximity effects of the heterojunction [74]. Figure 7a shows the sublattices of graphene on $\mathrm{EuO}$ represented with 
(a)

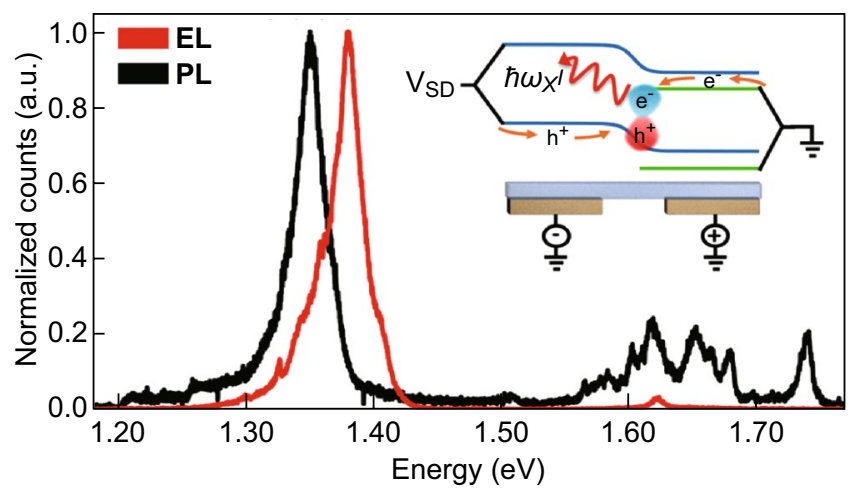

(b)
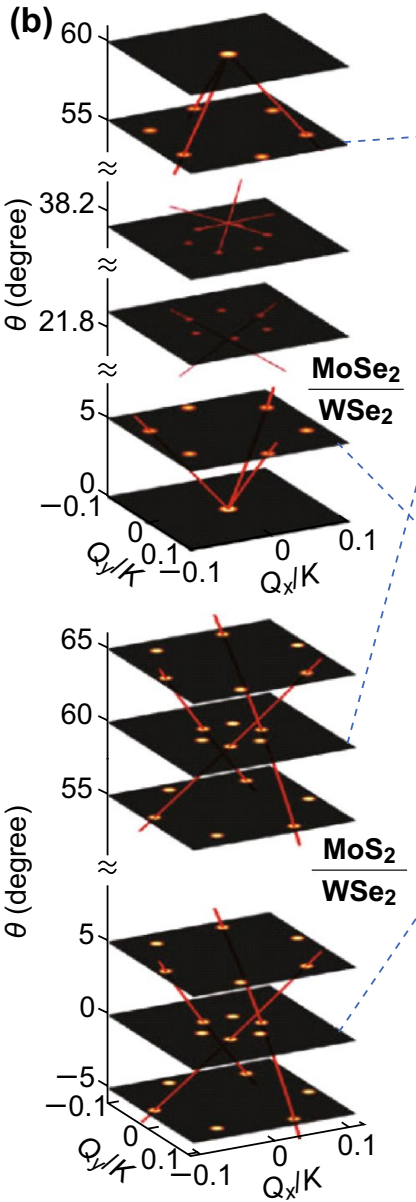
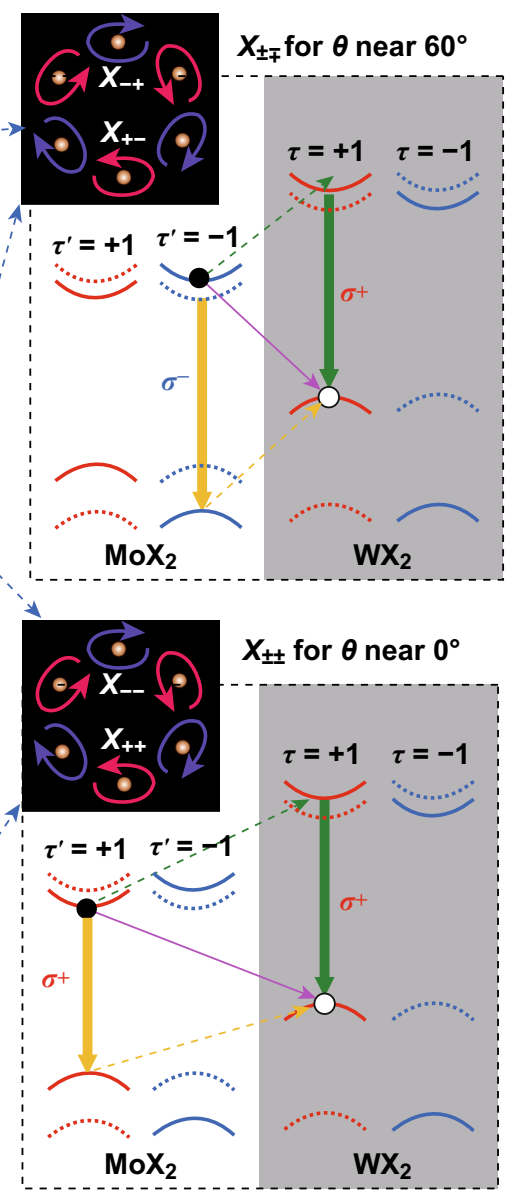

(c)

(d)
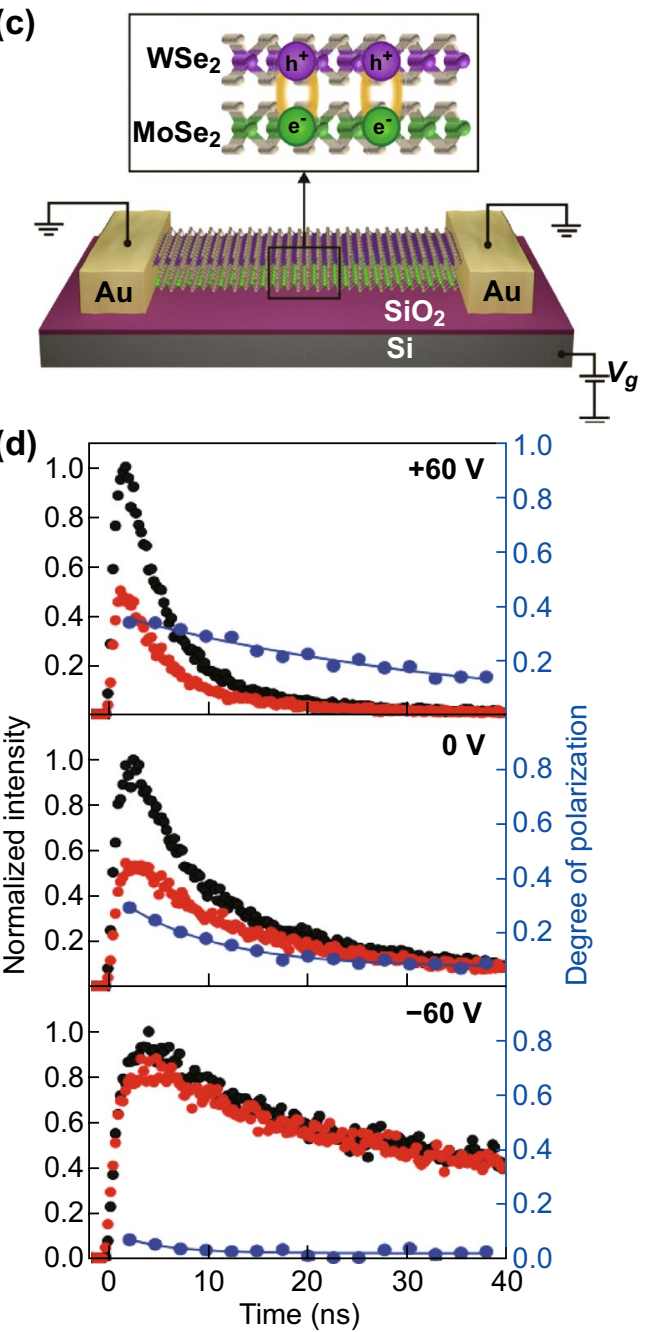

(e)
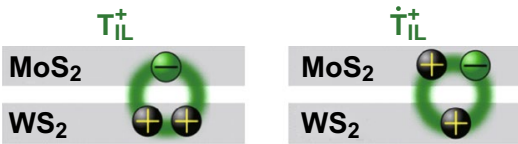

$\mathrm{T}_{\mathrm{IL}}^{-}$

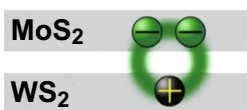

$\dot{\mathrm{T}}_{\mathrm{IL}}^{-}$

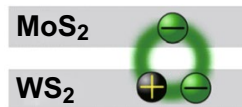

Fig. 6 The properties of excitons in 2D heterostructures. a EL spectra of the $\mathrm{MoSe}_{2} / \mathrm{WSe}_{2}$ vertical heterostructure, a small intralayer signal at $1.62 \mathrm{eV}$ was detected, and the inset illustrates the device structure. (Blue and green lines represent the $\mathrm{WSe}_{2}$ and $\mathrm{MoSe}_{2}$, respectively.) Reprinted with permission from Ref. [66]. b The optical selection rules in a $\mathrm{MoX}_{2} / \mathrm{WX}_{2}$ heterostructure with twist angle $\theta$ of $0^{\circ}$ and $60^{\circ}$, respectively. Reprinted with permission from Ref. [17]. c, d Time-resolved PL (d) of $\mathrm{WSe}_{2} / \mathrm{MoSe}_{2}$ heterostructure (c) after $\sigma+$ pulsed laser excitation, black dots and red dots corresponding to the co-polarized emission $(\sigma+)$ and cross-polarized emission $(\sigma-)$, and the blue line shows the degree of polarization, which is tunable by the gate voltage. Reprinted with permission from Ref. [67]. e The trions composed in different ways existing in the $\mathrm{MoS}_{2} / \mathrm{WS}_{2}$ bilayer. Reprinted with permission from Ref. [68]. (Color figure online) 
(a)

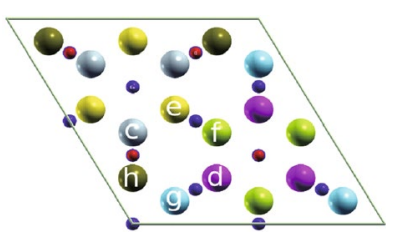

(b)

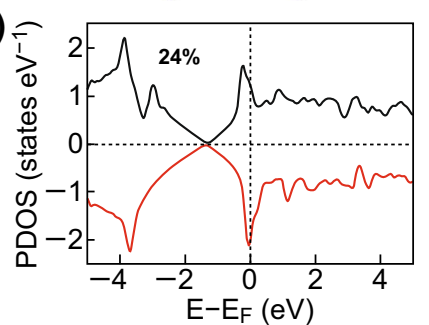

(e)

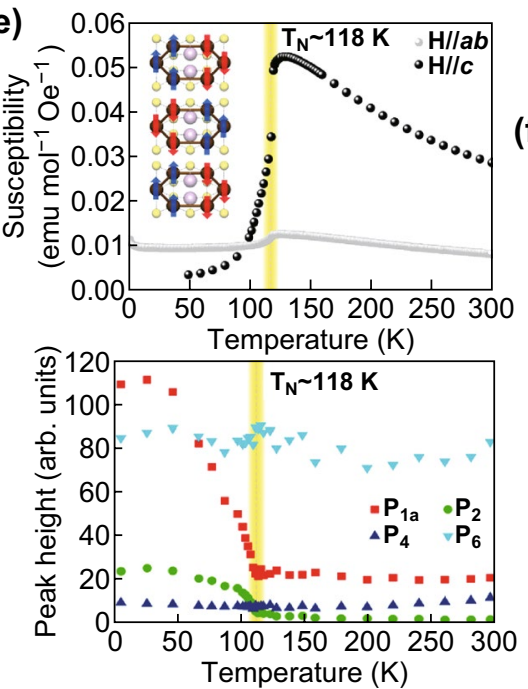

(h)

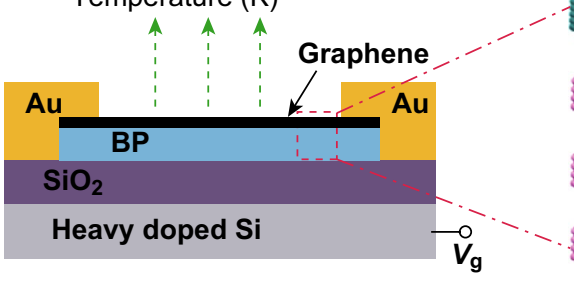

(c)

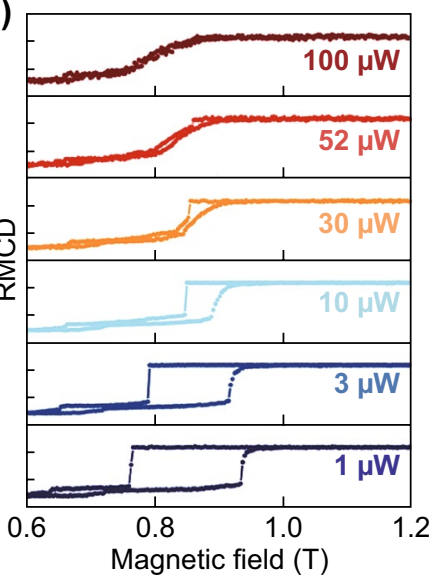

(d)

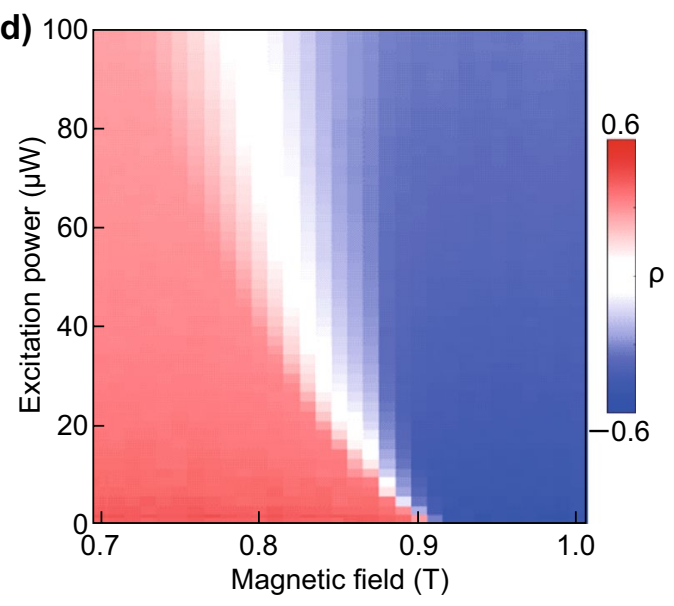

(f)

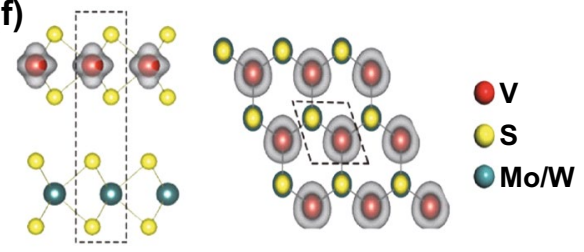

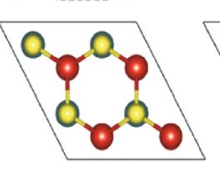
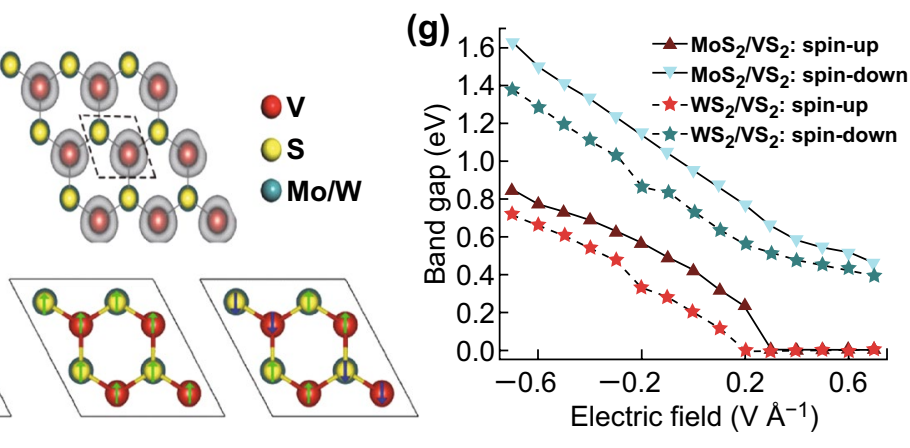

Monolayer G
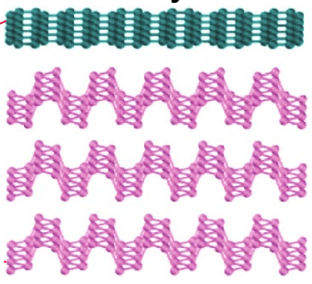

Multilayer BP

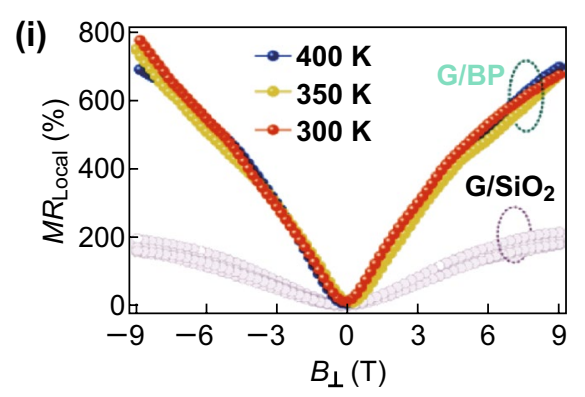

Fig. 7 Magnetic properties of 2D heterostructures. The broken lattice with six sublattices of graphene on the EuO (a) and the calculated spin polarization in graphene layer (b). Reprinted with permission from Ref. [74]. The RMCD (c) and polarization $\rho(\mathbf{d})$ of $\mathrm{WSe}_{2} / \mathrm{CrI}_{3}$ heterostructure under sweeping external magnetic field and different excitation power. Reprinted with permission from Ref. [75]. e The transition of several PL peaks and the magnetic susceptibility along the out-of-plane direction (black) in $\mathrm{FePS}_{3}$ as the temperature increases. Reprinted with permission from Ref. [77]. The spin density and possible magnetic ground state (NM, FM, AFM) of $\mathrm{XS}_{2} / \mathrm{VS}_{2}$ heterostructures (f) and the dependence of band gap (g) and electrical field, and the calculated magnetic moment and relative energy is listed in the picture. Reprinted with permission from Ref. [78]. Schematic diagram of the device based on graphene/multilayer BP heterostructure (h) and the huge MR (i) compared with graphene on $\mathrm{SiO}_{2}$ substrate. Reprinted with permission from Ref. [79]

different colors and letters. Due to the existence of the EuO substrate, the two sublattices of isolated graphene break into six folders as shown in Fig. 7b. Such structural change resulting from the proximity effect will enhance the magnetic moment of surface Eu atoms, causing variable spin polarizations on the graphene sublattices with a calculated spin polarization of about $24 \%$ in average, and change the band structure of graphene. The recent research suggests that such magnetic exchange field (MEF) can be tuned over a range of $20 \mathrm{~T}$ by small changes in the laser excitation 
power [75]. Figure 7c shows the magnetization of $\mathrm{CrI}_{3}$ in a monolayer $\mathrm{WSe}_{2} /$ multilayer $\mathrm{CrI}_{3}$ heterostructure probed via reflection magnetic circular dichroism (RMCD) as a function of the external magnetic field at different excitation light power. The RMCD exhibits a very similar power-dependent hysteresis loop behavior. Such opto-magnetic effect enables the power-switchable valley properties as illustrated in the PL spectra in Fig. 7d, where the polarization $\rho$ (defined as $\left(I_{+}-I_{-}\right) /\left(I_{+}-I_{-}\right)$, with $I_{ \pm}$being the PL peak intensity excited by $\sigma_{ \pm}$polarized laser) flips in sign at certain external magnetic field with the increasing of the excitation power.

The search of suitable 2D magnetic materials remains a challenge at the moment. The $\mathrm{CrI}_{3}$ mentioned above is layered magnetic material whose magnetic properties are strongly related to the number of layers [76]. Besides, a new class of magnetic heterostructure materials, called transition metal phosphorus trichalcogenides (TMPS, TM=V, Mn, Fe, $\mathrm{Co}, \mathrm{Ni}$, or $\mathrm{Zn}$ ), could also be easily exfoliated and their magnetic ground state is strongly depended on the TM element. An Ising-type antiferromagnetic ordering from bulk to the monolayer has been reported for $\mathrm{FePS}_{3}$, a TMPS material. The Raman peaks (Fig. 7e) show a transition at the Neel temperature $\left(T_{\mathrm{N}}\right)$ of $118 \mathrm{~K}$ [77].

The $T_{\mathrm{C}}$ of ferromagnetic materials in the above heterostructures is too low for them to be applied at room temperature. Current theoretical calculations have shown that $\mathrm{XS}_{2} / \mathrm{VS}_{2}$ heterostructures are ferromagnetic and are expected to have ultrahigh $T_{\mathrm{C}}$ [78]. Figure $7 \mathrm{f}$ shows the optimized configurations, local magnetic arrangements, magnetic moments and energy relative to that of the ferromagnetic configuration for non-magnetic (NM), ferromagnetic (FM), and antiferromagnetic (AFM) states, respectively. Clearly, the FM state is the most stable one for the two heterostructures. The $\mathrm{XS}_{2} / \mathrm{VS}_{2}$ heterostructures have the FM ground states in theory, and their $T_{\mathrm{C}}$ can be calculated by the following equation:

$\gamma k_{\mathrm{B}} T_{\mathrm{C}} \ominus / 2=E_{\mathrm{AFM}}-E_{\mathrm{FM}}$

where $\gamma$ is the dimension of the $\mathrm{XS}_{2} / \mathrm{VS}_{2}$ system, $k_{\mathrm{B}}$ is the Boltzmann constant, and $E_{\mathrm{AFM}}$ and $E_{\mathrm{FM}}$ are the energies of the unit-cell system with AFM and FM coupling, respectively. The obtained very high $T_{\mathrm{c}}$ values are $485 \mathrm{~K}$ for the $\mathrm{MoS}_{2} / \mathrm{VS}_{2}$ heterostructure and $487 \mathrm{~K}$ for the $\mathrm{WS}_{2} / \mathrm{VS}_{2}$ heterostructure. Interestingly, a semiconductor-metal transition occurred under the modulation of the external electric field (Fig. 7 g), indicating a potential for generating pure spinpolarized currents. Moreover, many other 2D magnetic materials have been studied experimentally or theoretically as shown in Table 2. Most of the 2D magnetic materials are in the form of $\mathrm{MX}(\mathrm{M}=\mathrm{Cr}, \mathrm{Co}, \mathrm{V}, \mathrm{Mo}, \mathrm{Mn}$, etc., and $\mathrm{X}=\mathrm{C}$, $\mathrm{O}, \mathrm{S}, \mathrm{Se}$ or $\mathrm{N})$ or $\operatorname{MAX}(\mathrm{M}=\mathrm{Cr}$ or $\mathrm{Fe} ; \mathrm{A}=\mathrm{Ge}, \mathrm{Si}, \mathrm{Al}, \mathrm{Sn}$, etc., and $\mathrm{X}=\mathrm{Te}$ ), and most of them are semiconductors.

Table 2 The calculated or experimental properties of different 2D magnetic materials

\begin{tabular}{|c|c|c|c|c|c|c|}
\hline Material & $T_{\mathrm{c}}(\mathrm{K})$ & $\begin{array}{l}\text { Saturation magnetiza- } \\
\text { tions }\left(\mu_{\mathrm{B}} / \text { unit) }\right.\end{array}$ & Band gap $(\mathrm{eV})$ & Exchange parameters & Kerr rotation angle & References \\
\hline $\mathrm{VSe}_{2} \mathrm{ML}$ & $>330 \mathrm{~K}$ & 15 & $55 \mathrm{~m}(15 \mathrm{~K})$ & - & - & [80] \\
\hline $\mathrm{Fe}_{3} \mathrm{GeTe}_{2}$ & $\begin{array}{l}207 \mathrm{~K} \text { (bulk) } \\
130 \mathrm{~K} \text { (ML) }\end{array}$ & - & - & - & - & [81] \\
\hline $\left.\mathrm{TL} \mathrm{Fe}_{3} \mathrm{GeTe}_{2}\right]$ & $\sim 300 \mathrm{~K}\left(V_{\mathrm{g}}=1.75 \mathrm{~V}\right)$ & 1.8 & - & $J_{i j}=10 \mathrm{mV}($ Heisenberg model $)$ & - & [82] \\
\hline$* \mathrm{CrC} \mathrm{ML}$ & $>330 \mathrm{~K}$ & 8 & 2.85 & $\begin{array}{l}J_{1}=7.4 \mathrm{meV} J_{2}=14.7 \mathrm{meV} \\
\quad(\text { Heisenberg model) }\end{array}$ & - & [83] \\
\hline$* \mathrm{MnO}_{2} \mathrm{ML}$ & $\begin{array}{l}140 \mathrm{~K} \\
210 \mathrm{~K} \text { (strained) }\end{array}$ & $3(<75 \mathrm{~K})$ & 3.41 & $J=1.72 \mathrm{meV}$ (Ising model) & - & [84] \\
\hline$* \mathrm{Co}_{2} \mathrm{~S}_{2} \mathrm{ML}$ & $>404 \mathrm{~K}$ & & - & $\begin{array}{l}J_{1}=58.7 \mathrm{meV} J_{2}=15.8 \mathrm{meV} \\
\quad \text { (Ising model) }\end{array}$ & - & [85] \\
\hline $\mathrm{GdAg}_{2}$ & $85 \mathrm{~K}$ & $5(5 \mathrm{~K})$ & - & - & - & [86] \\
\hline$* \mathrm{MnS}_{2} \mathrm{ML}$ & $225 \mathrm{~K}, 330 \mathrm{~K}$ (strained) & 3 & 0.69 & - & - & [87] \\
\hline$* \mathrm{MnSe}_{2} \mathrm{ML}$ & $250 \mathrm{~K}, 375 \mathrm{~K}$ (strained) & 3 & 0.01 & - & - & [87] \\
\hline $\mathrm{CrI}_{3}$ & $\begin{array}{l}61 \mathrm{~K} \text { (bulk) } \\
45 \mathrm{~K} \text { (ML) } \\
\text { AFM (BL) }\end{array}$ & 3 & - & - & $5 \pm 2 \mathrm{mrad}$ at $\mu_{\mathrm{oH}}=0 \mathrm{~T}$ & [76] \\
\hline$* \mathrm{MoS}_{2} / \mathrm{VS}_{2} \mathrm{HS}$ & $485 \mathrm{~K}$ & 14.6 & Tunable & - & - & [51] \\
\hline$* \mathrm{WS}_{2} / \mathrm{VS}_{2} \mathrm{HS}$ & $487 \mathrm{~K}$ & 14.7 & Tunable & - & - & [51] \\
\hline
\end{tabular}

Theoretically predicted materials are marked by (*). The ML, BL, TL, and HS are short form of monolayer, bilayer, and heterostructure, respectively 
Furthermore, a magnetoresistance (MR) effect has been detected in some graphene-based heterostructures due to the interface state in the heterostructures. Liu et al. [79] fabricated a highly stable monolayer graphene/black phosphorus (Gra/BP) heterostructure device (Fig. 7h) that exhibits a giant MR (defined as $\frac{[R(B)-R(0)]}{R(0)} \times 100 \%$ ) of $775 \%$ (Fig. 7i), and the nonlocal MR more than $10,000 \%$ in the Gra/BP device at room temperature due to an enhanced flavor Hall effect induced by the BP channel. Those experimental results provide valuable information for the study of magnetization dynamics in devices such as magnetoresistive randomaccess memories.

\section{Applications in Devices}

\subsection{Electronic Devices}

2D materials have been extensively explored as channel materials for future electronic device applications because of their atomically thin channels that offer ideal electrostatic control to enhance the immunity to the short channel effects
[88] and the deprivation of band-edge sharpness resulting from chemical doping. The electronic devices based on the band engineering of 2D heterostructures have been widely reported.

Typically, the TFET usually uses an insulator thin layer of hBN as the dielectric layer, as well as TMDs with large band gap. Using 2D materials to replace traditional metallic oxides effectively avoid pinholes, oxygen doping, and interlayer defects [89]. The TFET based on the $\mathrm{SnSe}_{2} / \mathrm{WSe}_{2}$ vertical heterostructure (Fig. 8a) was fabricated with a subthreshold swing of $80 \mathrm{mV} \mathrm{dec}^{-1}$ and ultrahigh $I_{\mathrm{ON}} / I_{\mathrm{OFF}}$ ratio over $10^{6}$ (Fig. $8 \mathrm{~b}$ ), and such high performance can be realized simply by tuning the back-gate voltage to switch the BTBT effect [52].

Likewise, memories based on the floating-gate structure are also important applications of 2D heterostructures, where the selection of the floating-gate layer is crucial. To suppress the dark current in the device channel, gold nanoparticles (AuNPs) were selected to serve as the trapping layer [58]. Recently, researchers designed a programmable memory device based on a vertically stacked $\mathrm{MoS}_{2} / \mathrm{hBN} /$ (a)

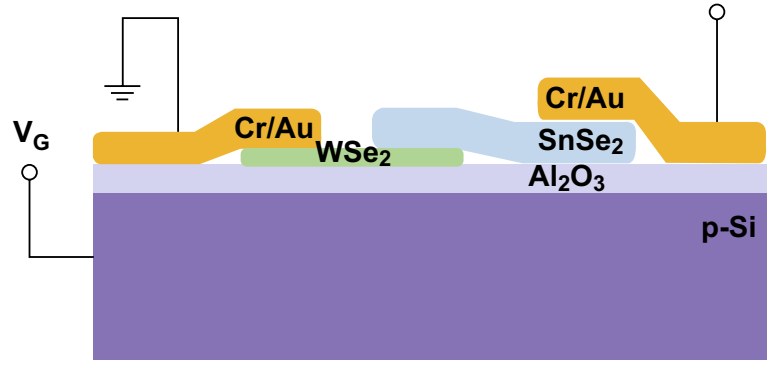

(c)

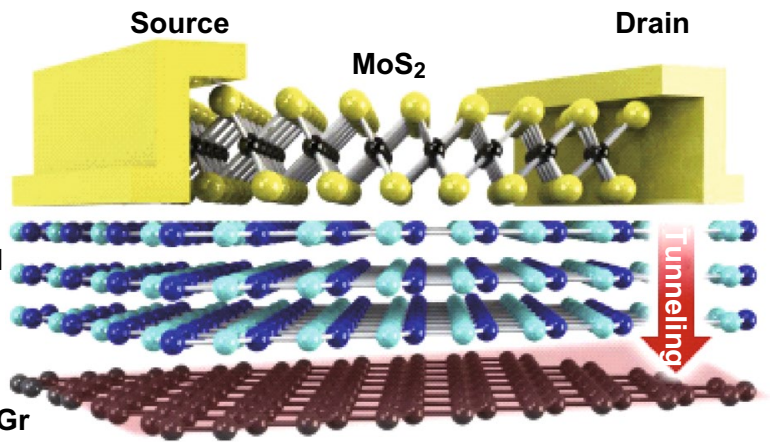

(b)

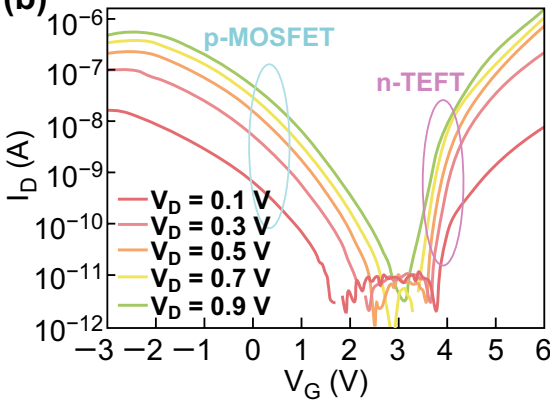

(d)

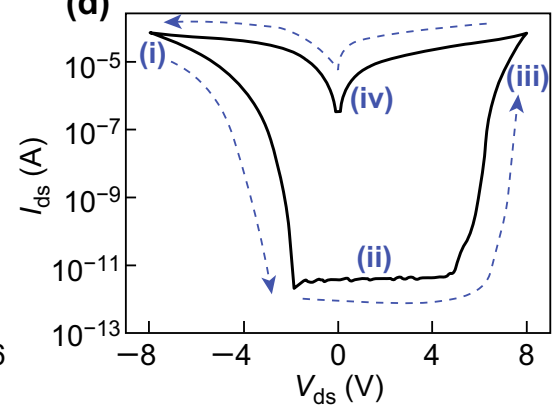

(e)

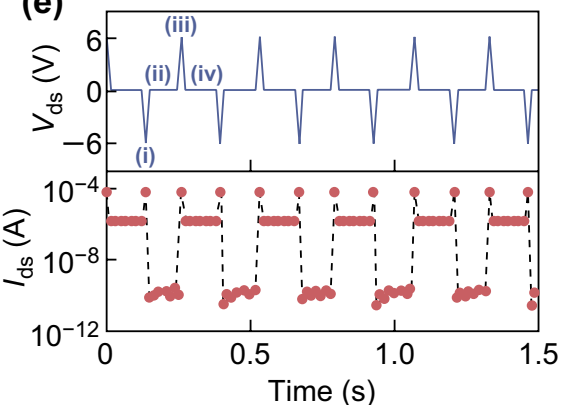

Fig. 8 Electronic devices based on 2D heterostructure. Transistor based on the BTBT in WSe $2 / \mathrm{SnSe}_{2}$ heterostructure (a) and its channel current (b) as a function of $V_{\mathrm{G}}$ at certain $V_{\mathrm{D}}$ from 0.1 to $0.9 \mathrm{~V}$. Reprinted with permission from Ref. [52]. Schematic diagram of programmable floatinggate memory based on $\mathrm{MoS}_{2} / \mathrm{hBN} /$ graphene heterostructure (c), the hysteresis loop of $I_{\mathrm{ds}}$ when $V_{\mathrm{ds}}$ sweeps forward and backward (d) and the corresponding $I_{\mathrm{ds}}$ when periodically repeat the four states by setting $V_{\mathrm{ds}}$ to -6 , 0 , and $+6 \mathrm{~V}$ (e). Reprinted with permission from Ref. [61] 
graphene heterostructure with graphene as the floating-gate [61] (Fig. 8c) which has ultrahigh on/off ratio and a high stretchability (>19\%). With the dielectric layer of hBN having an appropriate thickness, the on/off ratio over $10^{9}$ has been obtained. Figure $8 \mathrm{~d}$ shows the memory cycles realized by repeated voltage pulses as programming (1), reading (2), erasing (3), and reading (4) operations. Such functions can also be demonstrated in the hysteresis behavior in the $I_{\mathrm{ds}}-V_{\mathrm{ds}}$ plot (Fig. 8e) and are originated from the tunneling effect through hBN and the asymmetric potential drop caused by the highly resistivity of $\mathrm{MoS}_{2}$. Moreover, Si et al. [90] integrated 2D ferroelectric insulator of $\mathrm{CuInP}_{2} \mathrm{~S}_{6}$ on the top of $\mathrm{MoS}_{2}$ to build a nonvolatile memory device with a stable ferroelectric hysteresis loop in transfer characteristics.

\subsection{Optoelectronic Devices}

2D materials are outstanding candidates for optoelectronic devices due to their unique properties, including the wide response spectrum range, excellent flexibility, and strong light-matter interaction [91, 92]. Similarly, due to the generation of interlayer excitons and flexible band engineering, 2D heterostructures have been widely applied in optoelectronics including photodetectors [93, 94], photovoltaic devices, and light source devices [95-101]. Many experiments have shown that the photodetectors based on $2 \mathrm{D}$ heterostructures can be applied in a broad range of spectrum from ultraviolet (UV) to near-infrared (NIR), the same range as that of the photodetectors based on bulk materials, but wider than that of a single $2 \mathrm{D}$ heterostructure. Table 3 lists the main parameters of the latest photodetectors based on 2D heterostructures. Actually, the photodetectors based on $2 \mathrm{D}$ heterostructures perform better due to the generation of the strong built-in electrical field in such atomically thin structures [102]. Also, researchers are keeping exploring multi-ways to modulate devices and multi-structures to improve devices performance. It has been demonstrated that using graphene as the contact electrodes is an effective strategy to significantly increase the response speed (up to 5.5 ps Ref. [97]) of an atomically thin photodetector [95, $100,101]$, because graphene, as a 2D material with ultrahigh carrier mobility, can minimize the lateral diffusion in the semiconductor [103]. Recently, Tan et al. [42] built $\mathrm{WS}_{2} /$ $\mathrm{MoS}_{2}$ hetero-bilayer devices with layered graphene electrodes (Fig. 9a). By tuning the work function of graphene, the modification of the $\mathrm{SBH}$ at the interface of graphene and the TMD layer can be achieved. The lowering of the barrier as a result of the photogating effect would facilitate the re-injection of electrons into the TMD channel. Such devices displayed the highest photoresponsivity of up to $2340 \mathrm{~A} \mathrm{~W}^{-1}$ and a large internal photoconductive gain over $3.7 \times 10^{4}$, with an estimated specific detectivity of $4 \times 10^{11}$ Jones. Moreover, photodetectors based on $\mathrm{MoS}_{2}-\mathrm{WS}_{2}$ planar heterostructures [104] have also been reported to reach a detectivity of $4.36 \times 10^{13}$ Jones. The $\mathrm{p}-\mathrm{n}$ heterostructures are usually self-powered because the generated electron-hole pairs can get separated under built-in potential at zero bias. Compared with vertical heterostructures, the fabrication of in-plane junctions is much controllable and scalable due to the difficulties of controlling the stacking orientation. The extraordinary flexibility of heterostructures enabled the researchers to design a curved image sensor array based on a

Table 3 The performances of 2D-heterostructure-based photodetectors

\begin{tabular}{|c|c|c|c|c|c|c|}
\hline Heterostructures & Response spectrum & Responsivity & Detectivity (Jones) & Conditions & EQE & References \\
\hline $\mathrm{MoS}_{2}-\mathrm{GaTe}$ & - & $21.83 \mathrm{~A} \mathrm{~W}^{-1}$ & $8.4 \times 10^{13}$ & $V_{\mathrm{g}}=70 \mathrm{~V}$ & $61.68 \%$ & [106] \\
\hline $\mathrm{MoS}_{2} / \mathrm{Gra} / \mathrm{WSe}_{2}$ & $400-2400 \mathrm{~nm}$ & $10^{4} \mathrm{~A} \mathrm{~W}^{-1}$ (Vis) & $\begin{array}{l}10^{11} \text { (NIR) } \\
10^{14} \text { (Vis) }\end{array}$ & $\begin{array}{l}V_{\mathrm{ds}}=1 \mathrm{~V}, V_{\mathrm{g}}=0 \mathrm{~V} \\
P_{\mathrm{in}}=10^{-10} \mathrm{~W}\end{array}$ & $10^{6} \%$ & [107] \\
\hline $\mathrm{Gra} / \mathrm{MoS}_{2}$ & - & $5 \times 10^{8} \mathrm{~A} \mathrm{~W}^{-1}(300 \mathrm{~K})$ & - & $V_{\mathrm{ds}}=0.1 \mathrm{~V}, V_{\mathrm{g}}=-50 \mathrm{~V}$ & - & [108] \\
\hline $\mathrm{BP} / \mathrm{MoS}_{2}$ & - & $2.17 \mathrm{~A} \mathrm{~W}^{-1}$ & - & $V_{\text {bias }}=0 \mathrm{~V}$ & - & [109] \\
\hline $\mathrm{MoS}_{2}-\mathrm{WS}_{2}$ planar HS & - & $4.36 \mathrm{~mA} \mathrm{~W}^{-1}$ & $4.36 \times 10^{13}$ & $V_{\text {bias }}=0 \mathrm{~V}$ & $1.02 \%$ & [104] \\
\hline Polydiacetylene/Gra & UV to visible light & $556 \mathrm{~A} \mathrm{~W}^{-1}$ & $6 \times 10^{11}$ & $V_{\mathrm{ds}}=1 \mathrm{~V}, V_{\mathrm{g}}=0 \mathrm{~V}$ & - & [110] \\
\hline $\mathrm{Gra} / \mathrm{WTe}_{2}$ & - & $8.7 \mathrm{~A} \mathrm{~W}^{-1}$ & - & $V_{\mathrm{ds}}=0.5 \mathrm{~V}$ & $165 \%$ & [111] \\
\hline $\mathrm{WS}_{2} / \mathrm{MoS}_{2}$ & - & $1173 \mathrm{~A} \mathrm{~W}^{-1}$ & $4.1 \times 10^{11}$ & $V_{\mathrm{ds}}=10 \mathrm{~V}, V_{\mathrm{g}}=0 \mathrm{~V}$ & - & [42] \\
\hline InSe-Gra & $400-1000 \mathrm{~nm}$ & $60 \mathrm{~A} \mathrm{~W}^{-1}$ & $2.5 \times 10^{12}$ & $V_{\mathrm{ds}}=10 \mathrm{~V}, V_{\mathrm{g}}=0 \mathrm{~V}$ & $14,850 \%$ & [95] \\
\hline Gra/p-GaSe/n-InSe/Gra & $270-920 \mathrm{~nm}$ & $350 \mathrm{~A} \mathrm{~W}^{-1}$ & $3.7 \times 10^{12}$ & $V_{\mathrm{ds}}=2 \mathrm{~V}, V_{\mathrm{g}}=0 \mathrm{~V}$ & - & {$[100]$} \\
\hline
\end{tabular}


(a)

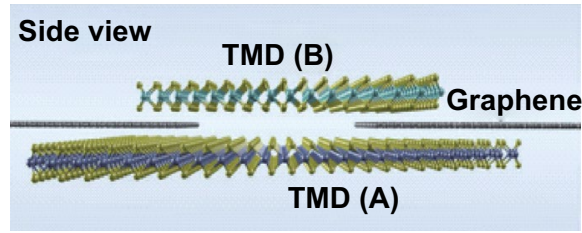

(b)
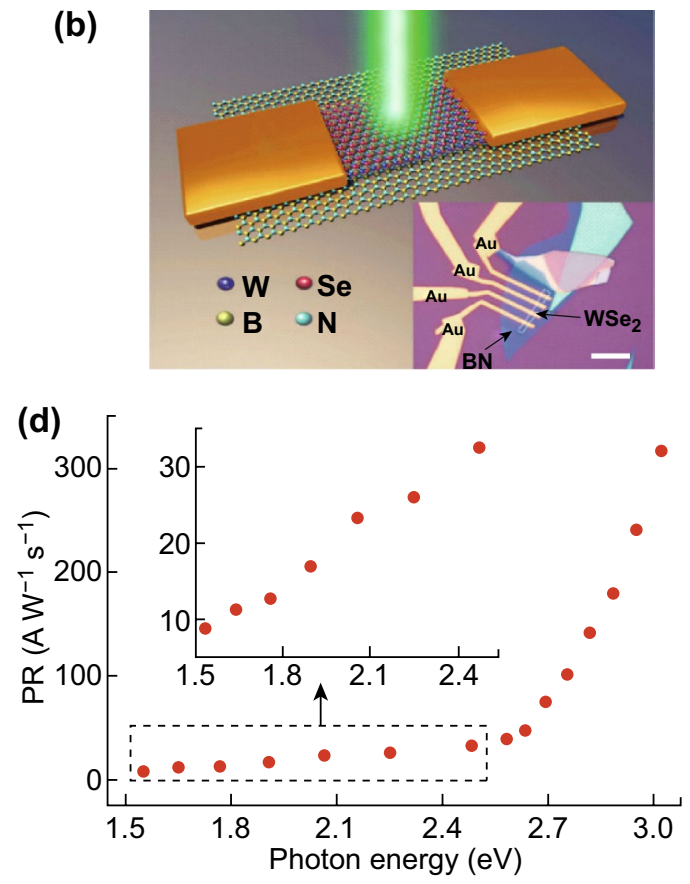

(e)

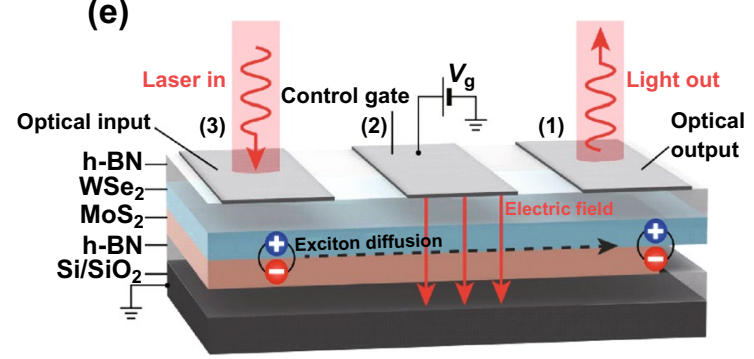

(c)
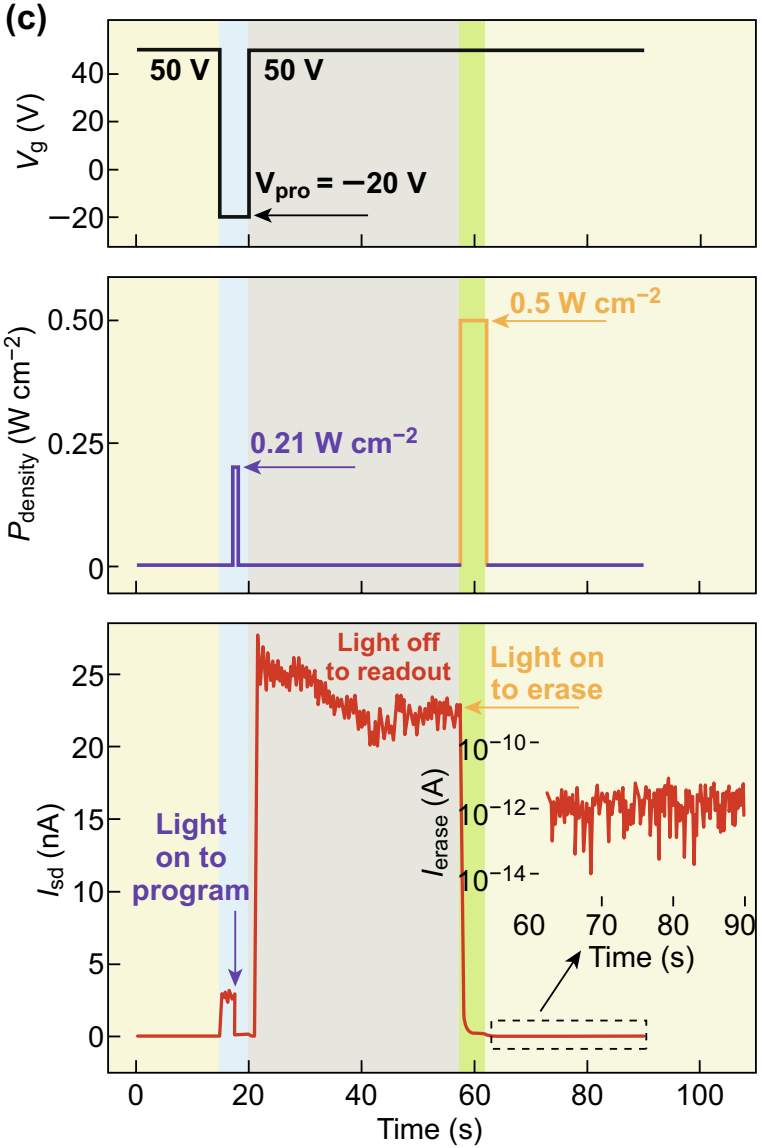

(f)

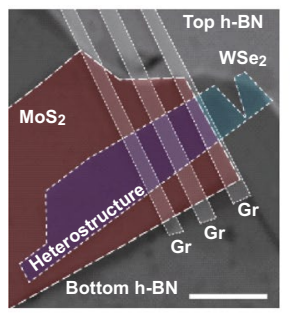

(g)

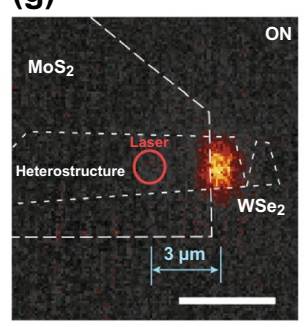

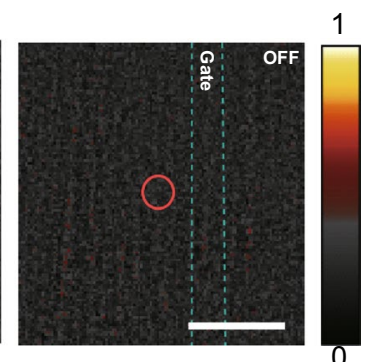

Fig. 9 2D heterostructures in optoelectronic applications. a Schematic diagram of TMD/TMD heterostructure with a graphene contact. Reprinted with permission from Ref. [42]. Schematic and optical picture of optoelectronic memory based on hBN/WSe $\mathrm{H}_{2}$ heterostructure (b) and the modulation of programming, reading and erasing state by $V_{\mathrm{g}}$ and incident illumination (c), and the wavelength-dependent PR (d). Reprinted with permission from Ref. [112]. Schematic and optical picture (e, f) of the multi-gate exciton device. The excitation region is marked by the red circle, and the recombination of the interlayer excitons occurs at the edge of the heterostructure. The modulation of exciton flux by $V_{\mathrm{g}}$ at room temperature is illustrated in diagram (g). Reprinted with permission from Ref. [113]

$\mathrm{MoS}_{2}$-graphene heterostructure [105] which can be applied as a human eye-inspired soft implantable optoelectronic device for detecting optical signals through programmed electrical stimulation to optic nerves.

Moreover, the optoelectronic memories based on 2D heterostructures have been designed which can accumulate and release photo-generated carriers under an electric field and light irradiation. In addition, the introduction of 2D materials makes it possible to realize miniature, flexible, and lowenergy-consumption optoelectronic storage [105]. Recently, Xiang et al. [112] successfully fabricated a multi-bit nonvolatile optoelectronic memory based on a stacked $\mathrm{WSe}_{2} / \mathrm{hBN}$ heterostructure as shown in Fig. 9b, which is also a filter-free color image sensor. The device can be programmed to read 
and erase by adjusting the gate voltage and the light pulse. The corresponding band alignment is shown in Fig. 9. The positive charges can be stored in $\mathrm{hBN}$ even after removing the negative gate and switching off the light (Fig. 9c). The device has performed a retention time over $4.5 \times 10^{4} \mathrm{~s}$ and possesses over 128 (7 bit) storage states. Interestingly, the storage states at different wavelengths are highly distinct (Fig. 9d), indicating excellent wavelength distinguishing capability of the $\mathrm{WSe}_{2} / \mathrm{hBN}$ optoelectronic memory.

In addition, the reduced Coulomb screening in the atomically thin crystals led to a dramatic increase in the exciton binding energy and therefore stabilized these excitons at room temperature. Recently, Unuchek et al. [113] reported excitonic devices made of $\mathrm{MoS}_{2} / \mathrm{WSe}_{2}$ van der Waals heterostructures encapsulated in hBN (Fig. 9e) demonstrating electrically controlled multi-gate transistor actions at room temperature. The interlayer exciton was excited in the heterostructure, and the recombination was successfully observed at the edge of the heterostructure (Fig. 9g). The device was switched by adjusting the $V_{\mathrm{g} 1}$, reaching an on/off ratio of 100. Furthermore, under the modulation of the bias voltage, the excitons would drift (forward bias voltage) to the low potential or be limited in the potential hydrazine (reverse bias voltage), so the diffusion distance of the exciton could be adjusted to reach $5 \mu \mathrm{m}$ under a forward bias voltage.

\subsection{Spintronic and Valleytronic Devices}

2D materials have excellent spin-valley properties; for example, graphene exhibits outstanding electrical, thermal, and mechanical properties. It also displays a very long spin diffusion length up to room temperature that facilitates the spin injection, manipulation, and detection in an integrated device leading to the realization of scalable and ultrafast nonvolatile logic circuits with ultralow energy dissipation. More recently, the spin valve effect in FM/2D-material/ FM sandwich-like magnetic junction has been observed [114-117]. Particularly, in certain FM/G/FM junctions $(\mathrm{FM}=\mathrm{Ni}$, Co etc.), the lattice mismatch between graphene and FM is very small, and simultaneously, a spin filtering effect is theoretically permitted. As shown in Fig. 10a [118], only the spin states at Dirac point in FM can be injected into graphene and the calculation indicates that only minority spins exist at the Dirac point. The introduction of 2D heterostructures in magnetic tunneling junctions can efficiently modulate the result of the spin filter due to the different spin polarizations at different interfaces. Iqbal et al. [119] successfully observed the negative tunnel magnetoresistance (TMR) of $-0.85 \%$ in a NiFe/G-hBN/Co magnetic junction under room temperature (Fig. 10b). However, the quality of the interfaces and the lattice orientation between different layers are very crucial.

Besides, monolayer TMDs with a broken inversion symmetry own two degenerated inequivalent valleys that are related by time-reversal symmetry [120]. This property and the strong spin-orbit coupling are responsible for the unique physics in TMDs, especially the coupled spin and valley of freedom. With a direct band gap, TMDs offer the opportunity to excite carriers selectively within a particular valley with a specific valley pseudospin using circularly polarized light. Also, the valley Hall effects can be observed in a certain doped TMD sample [121, 122].

However, the external control of valleytronic devices remains a challenge because the conditions of lifting the valley degeneracy by Zeeman splitting in a single $2 \mathrm{D}$ material are very demanding that usually require strong magnetic field and low temperature [123], while tailoring graphene magnetic properties by structural engineering such as doping [10] and defects [124] inevitably increase the complexity in operations.

Utilizing the interfacial magnetic exchange field (MEF) from a ferromagnetic substrate that greatly enhanced valley splitting in monolayer TMDs has been found recently. Chuan Zhao et al. [125] successfully put the $\mathrm{WSe}_{2}$ monolayer on $\mathrm{Si} / \mathrm{SiO}_{2}$ and on ferromagnetic EuS substrates. The magnetic field was perpendicular to the plane (Fig. 10c). In $\mathrm{WSe}_{2}$ on the $\mathrm{SiO}_{2}$ substrate, the $\Delta E$ was $1.5 \mathrm{meV}$ at $7 \mathrm{~T}$ and $7 \mathrm{~K}$. But in $\mathrm{WSe}_{2}$ on the EuS, the valley splitting reached $3.9 \mathrm{meV}$. Figure 10d, e shows the measurements of the systematic field and temperature dependences of the splitting. With the field increased further, the rate of $\Delta E_{\mathrm{ex}}$ increase got slower and then tended to saturation at high magnetic fields. Also, with the increase in temperature, $\Delta E_{\mathrm{ex}}$ decreased accordingly. Such behaviors are very similar to the field- and the temperature-dependent magnetization of EuS.

Another thought of combining is to select another material whose properties can compensate for the setbacks of the material. Typically, for the graphene and TMDs in spintronics [127], the long-distance spin transport capability of graphene has been demonstrated at 
(a)
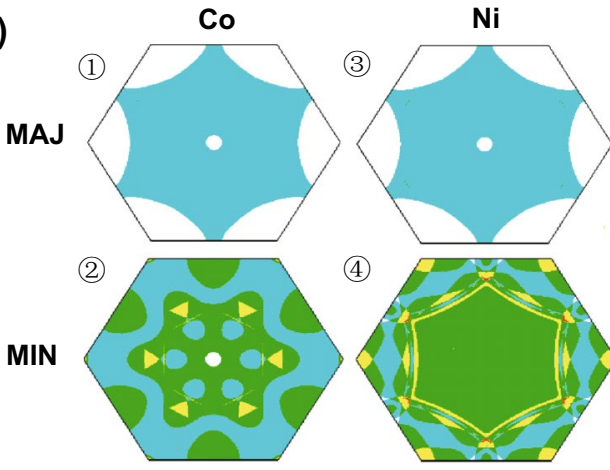

(4)

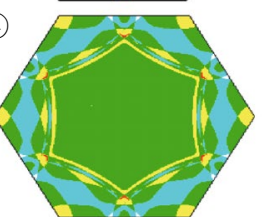

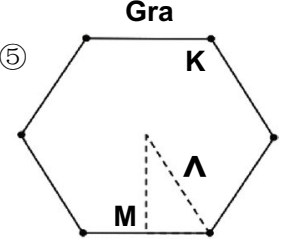

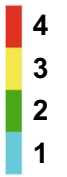

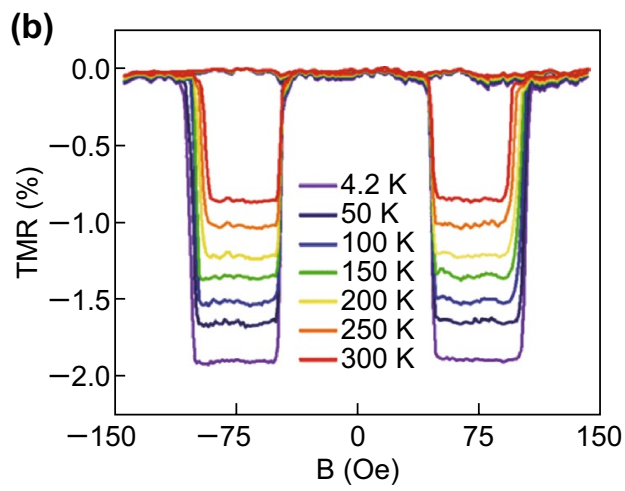

(e) (c)

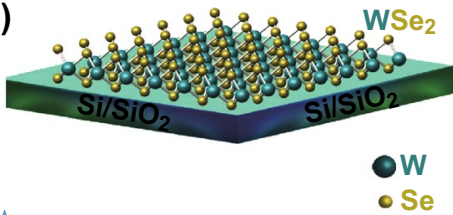

$B \uparrow$

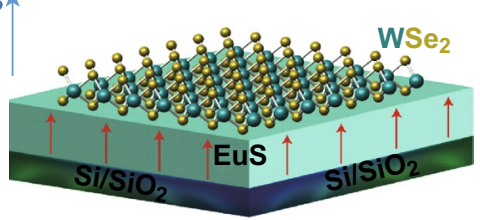

(f)

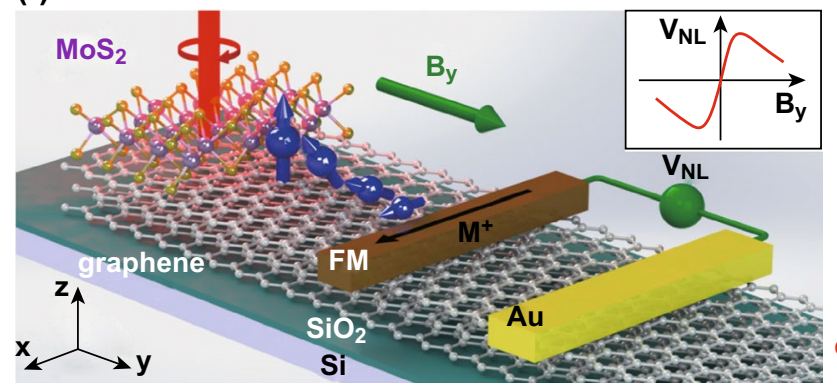

(d)

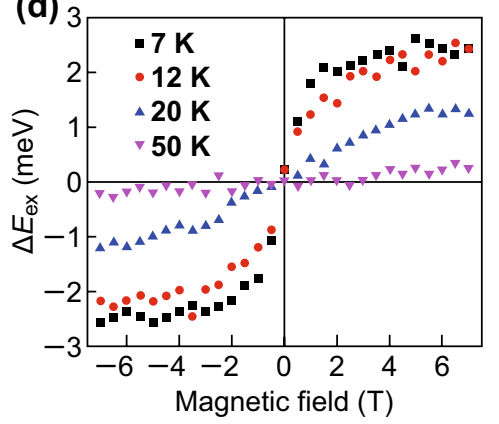

(g)

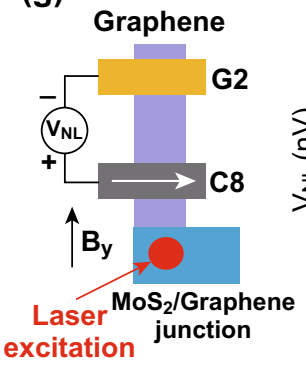

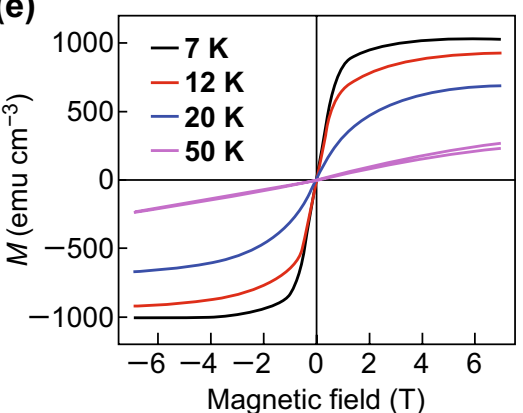

(h)

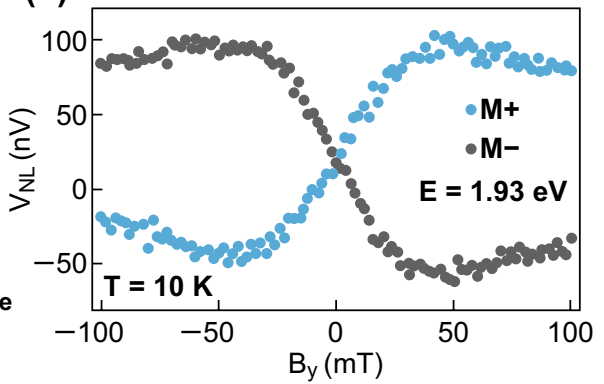

Fig. 10 Spintronic and valleytronic applications of 2D heterostructures. a The calculated majority and minority spin states of Co (11-(2) and Ni (3)-4) and the Fermi surface of graphene (5), and the color bar indicates the number of Fermi surface sheets. Reprinted with permission from Ref. [118]. b TMR of the NiFe/Gra-hBN/Co magnetic junction at a different temperature. Reprinted with permission from Ref. [119]. WSe 2 on the $\mathrm{SiO}_{2}$ substrate and the EuS substrate (c), and the valley splitting (d) and magnetization (e) in the WSe 2 /EuS structure under sweeping external vertical magnetic field at different temperatures. Reprinted with permission from Ref. [125]. Schematic diagram of MoS $/$ graphene heterostructure and the Hanle measurement $(\mathbf{f}, \mathbf{g})$ and the Hanle signal (h) detected after the laser excitation on the heterostructure region at a temperature of $10 \mathrm{~K}$. Reprinted with permission from Ref. [126]. (Color figure online)

room temperature [128], but the lack of the SOC made it complicated to generate pure spin current. In contrast, spin/valley polarization can be efficiently generated in monolayer TMDs such as $\mathrm{WS}_{2}$ and $\mathrm{MoS}_{2}$ via external excitation field. Recently, lateral spin or valley transport has been realized at room temperature by fabricating $\mathrm{MoS}_{2}$ /few-layer graphene hybrid spin valves as shown in Fig. 10f. Luo et al. [126] fabricated the monolayer $\mathrm{MoS}_{2} /$ few-layer-graphene hybrid spin valves and successfully injected the spin signal generated by circularly polarized light excitation on the TMD layer into the graphene layer. Figure 10g shows the illustration of optical spin injection, lateral spin transport, and electrical spin detection in a monolayer $\mathrm{MoS}_{2}$ /few-layer-graphene hybrid spin valve structure. Figure $10 \mathrm{~h}$ shows the electrical spin signal $V_{\mathrm{NL}}$ as a function of external magnetic field $B_{\mathrm{y}}$ under $T=10 \mathrm{~K}$. The spin signal can still be easily detected at room temperature but it's about 5 times smaller due to the increased intervalley scattering which reduces the valley polarization in monolayer $\mathrm{MoS}_{2}$. 


\section{Summary and Outlook}

It is clear that remarkable progress has been achieved in the study of $2 \mathrm{D}$ heterostructures, such as the development in realizing controllable CVD growth of planar heterostructures, the distinctive properties of magnetism and spatially separated excitons, as well as the various design and effective modulation of devices. These advances are fundamental bases for future scalable applications of 2D heterostructures. Additionally, 2D heterostructures can display unique physics phenomena due to the coupling effect and the electronic transport at the junction, offering an ideal platform for fundamental research in physics.

Nevertheless, there are still great challenges in the research of 2D heterostructures. At the first, successful synthesis of vertical heterostructures by CVD has been rare and more often the stacked heterostructures were fabricated by artificial transfer that needed a proper annealing process, a procedure that could cause damage to the samples. Thus, the priority is to develop new strategies for scalable and controllable fabrication of 2D heterostructures with high-quality interfaces and certain stack orientation, while the CVD synthesis is an ideal method its gas system needs to be optimized to for better control of the reaction conditions. As for deterministic transfer method, in-depth studies on surface physical chemistries are needed for the invention of new types of transfer carriers. Secondly, new materials, such as layered magnetic materials with high $T_{\mathrm{C}}$, need to be created, which will enable a lot of new combinations of 2D heterostructures. Thirdly, to modulate the magnetic properties, band structure, and charge transport characteristics of 2D heterostructures, conducting external electric field, optical field, magnetic field, strain treatment, together with the structural and surface engineering on the materials, will be meaningful and helpful research points. What's more, for the fabrication and design of devices, the selection of proper materials as the electrode contact for reducing the $R_{\mathrm{C}}$ remains a tough task. The structural design of new devices to include floating-gate, multi-gate, and array structures is also very challenging.

In order to further explore the properties and applications of 2D heterostructures, several opinions could be taken into consideration for future development. Firstly, the integration of layered ferromagnetic insulator and monolayer TMD will greatly enhance the valley splitting in TMD, so the synthesis of a layered ferromagnetic insulator material whose $T_{\mathrm{C}}$ is over the room temperature could enable the development of valley-storage or valleylogical devices. Secondly, mix-dimension devices based on 0D-2D, 1D-2D, or 3D-2D heterostructures are also an effective way to solve the current problems. By combining quantum dots, nanowire, nanoribbon, or waveguide structures [129] with 2D sheets, more novel properties, such as interfacial disorders, will be realized. Finally, the fabrication strategy needs further improvement. For the CVD method, modulation of gas flow direction and temperature is a key factor for controlled reaction. For the transfer method, the development of a new transfer media layer and an automatic strategy is helpful to realize scalable fabrication and high yield. Hence, the heterostructures of $2 \mathrm{D}$ materials are promising for exploring new physics in 2D materials and realizing functional applications. The future of 2D heterostructures is full of massive novel possibilities.

Acknowledgements Yanping Liu would like to acknowledge Ye $\mathrm{Yu}$ for useful discussions. This work was supported by NSF of China (Grant No. 61775241) and partly by the Innovation-driven Project (Grant No. 2017CX019) and Youth Innovation Team (Grant No. 2019012) of CSU. Z. W. L. acknowledges the funding support from the Australian Research Council (ARC Discovery Projects, DP180102976).

Open Access This article is distributed under the terms of the Creative Commons Attribution 4.0 International License (http:// creativecommons.org/licenses/by/4.0/), which permits unrestricted use, distribution, and reproduction in any medium, provided you give appropriate credit to the original author(s) and the source, provide a link to the Creative Commons license, and indicate if changes were made.

\section{References}

1. K.S. Novoselov, A.K. Geim, S.V. Morozov, D. Jiang, Y. Zhang, S.V. Dubonos, I.V. Grigorieva, A.A. Firsov, Electric field effect in atomically thin carbon films. Science 306(5696), 666-669 (2004). https://doi.org/10.1126/scien ce. 1102896

2. M. Osada, T. Sasaki, Two-dimensional dielectric nanosheets: novel nanoelectronics from nanocrystal building blocks. Adv. Mater. 24(2), 210-228 (2012). https://doi.org/10.1002/ adma.201103241

3. L.M. Malard, J. Nilsson, D.C. Elias, J.C. Brant, F. Plentz et al., Probing the electronic structure of bilayer graphene by 
Raman scattering. Phys. Rev. B 76(20), 201401 (2007). https ://doi.org/10.1103/PhysRevB.76.201401

4. V. Tran, R. Soklaski, Y.F. Liang, L. Yang, Layer-controlled band gap and anisotropic excitons in few-layer black phosphorus. Phys. Rev. B 89(23), 235319 (2014). https://doi. org/10.1103/PhysRevB.89.235319

5. K.F. Mak, C. Lee, J. Hone, J. Shan, T.F. Heinz, Atomically thin $\mathrm{MoS}_{2}$ : a new direct-gap semiconductor. Phys. Rev. Lett. 105(13), 136805 (2010). https://doi.org/10.1103/PhysRevLet t.105.136805

6. L. Xie, M.Z. Liao, S.P. Wang, H. Yu, L.J. Du et al., Graphene-contacted ultrashort channel monolayer $\mathrm{MoS}_{2}$ transistors. Adv. Mater. 29(37), 1702522 (2017). https://doi. org/10.1002/adma.201702522

7. Y.P. Liu, W.S. Lew, S. Goolaup, Z.X. Shen, L. Sun, T.J. Zhou, S.K. Wong, Observation of the semiconductor-metal transition behavior in monolayer graphene. Carbon 50(6), 22732279 (2012). https://doi.org/10.1016/j.carbon.2012.01.046

8. A. Allain, J.H. Kang, K. Banerjee, A. Kis, Electrical contacts to two-dimensional semiconductors. Nat. Mater. 14(12), 1195-1205 (2015). https://doi.org/10.1038/nmat4452

9. P.R.Y. Gangavarapu, P.C. Lokesh, K.N. Bhat, A.K. Naik, Towards barrier free contacts to n-type CNTFETS using graphene electrodes, in 2016 IEEE Nanotechnology Materials and Devices Conference (NMDC 2016). https://doi. org/10.1109/nmdc.2016.7777064

10. J. Du, C.X. Xia, W.Q. Xiong, X. Zhao, T.X. Wang, Y. Jia, Tuning the electronic structures and magnetism of twodimensional porous $\mathrm{C}_{2} \mathrm{~N}$ via transition metal embedding. Phys. Chem. Chem. Phys. 18(32), 22678-22686 (2016). https ://doi.org/10.1039/C6CP03210A

11. Y.P. Liu, S. Goolaup, W.S. Lew, I. Purnama, M.C. Sekhar, T.J. Zhou, S.K. Wong, Excitonic bandgap dependence on stacking configuration in four layer graphene. Appl. Phys. Lett. 103(16), 163108 (2013). https://doi.org/10.1063/1.4825263

12. C.R. Dean, A.F. Young, I. Meric, C. Lee, L. Wang et al., Boron nitride substrates for high-quality graphene electronics. Nat. Nanotechnol. 5(10), 722-726 (2010). https://doi. org/10.1038/nnano.2010.172

13. W.J. Jie, Z.B. Yang, G.X. Bai, J.H. Hao, Luminescence in 2D materials and van der waals heterostructures. Adv. Opt. Mater. 6(10), 1701296 (2018). https://doi.org/10.1002/ adom.201701296

14. K.S. Novoselov, A. Mishchenko, A. Carvalho, A.H.C. Neto, 2D materials and van der Waals heterostructures. Science 353(6298), 9439 (2016). https://doi.org/10.1126/scien ce.aac9439

15. O.L. Sanchez, D. Ovchinnikov, S. Misra, A. Allain, A. Kis, Valley polarization by spin injection in a light-emitting van der waals heterojunction. Nano Lett. 16(9), 5792-5797 (2016). https://doi.org/10.1021/acs.nanolett.6b02527

16. C.L. Tan, H. Zhang, Epitaxial growth of hetero-nanostructures based on ultrathin two-dimensional nanosheets. J. Am. Chem. Soc. 137(38), 12162-12174 (2015). https://doi. org/10.1021/jacs.5b03590
17. H.Y. Yu, Y. Wang, Q.J. Tong, X.D. Xu, W. Yao, Anomalous light cones and valley optical selection rules of interlayer excitons in twisted heterobilayers. Phys. Rev. Lett. 115(18), 187002 (2015). https://doi.org/10.1103/PhysRevLet t.115.187002

18. F. Haque, T. Daeneke, K. Kalantar-zadeh, J. Ou, Twodimensional transition metal oxide and chalcogenide-based photocatalysts. Nano-Micro Lett. 10, 23 (2018). https://doi. org/10.1007/s40820-017-0176-y

19. X. Zhou, X.Z. Hu, J. Yu, S.Y. Liu, Z.W. Shu et al., 2D layered material-based van der waals heterostructures for optoelectronics. Adv. Funct. Mater. 28(14), 1706587 (2018). https:// doi.org/10.1002/adfm.201706587

20. J.A. Leon, N.C. Mamani, A. Rahim, L.E. Gomez, M.A.P. Silva, G.M. Gusev, Transferring few-layer graphene sheets on hexagonal boron nitride substrates for fabrication of graphene devices. Graphene 25-35, 48096 (2014). https://doi. org/10.4236/graphene.2014.33005

21. A. Castellanos-Gomez, M. Buscema, R. Molenaar, V. Singh, L. Janssen, H.S.J. van der Zant, G.A. Steele, Deterministic transfer of two-dimensional materials by all-dry viscoelastic stamping. 2D Mater. 1(1), 011002 (2014)

22. P. Pedrinazzi, J.M. Caridad, D.M.A. Mackenzie, F. Pizzocchero, L. Gammelgaard et al., High-quality graphene flakes exfoliated on a flat hydrophobic polymer. Appl. Phys. Lett. 112(3), 033101 (2018). https://doi.org/10.1063/1.5009168

23. P.J. Zomer, S.P. Dash, N. Tombros, B.J. van Wees, A transfer technique for high mobility graphene devices on commercially available hexagonal boron nitride. Appl. Phys. Lett. 99(23), 232104 (2011). https://doi.org/10.1063/1.3665405

24. J.I.J. Wang, Y.F. Yang, Y.A. Chen, K. Watanabe, T. Taniguchi, H.O.H. Churchill, P. Jarillo-Herrero, Electronic transport of encapsulated graphene and $\mathrm{WSe}_{2}$ devices fabricated by pick-up of prepatterned hBN. Nano Lett. 15(3), 1898-1903 (2015). https://doi.org/10.1021/nl504750f

25. R. Frisenda, E. Navarro-Moratalla, P. Gant, D.P. De Lara, P. Jarillo-Herrero, R.V. Gorbachev, A. Castellanos-Gomez, Recent progress in the assembly of nanodevices and van der Waals heterostructures by deterministic placement of 2D materials. Chem. Soc. Rev. 47(1), 53-68 (2018). https://doi. org/10.1039/c7cs00556c

26. M.A. Meitl, Z.T. Zhu, V. Kumar, K.J. Lee, X. Feng et al., Transfer printing by kinetic control of adhesion to an elastomeric stamp. Nat. Mater. 5(1), 33-38 (2006). https://doi. org/10.1038/nmat1532

27. Y.J. Gong, J.H. Lin, X.L. Wang, G. Shi, S.D. Lei et al., Vertical and in-plane heterostructures from $\mathrm{WS}_{2} / \mathrm{MoS}_{2}$ monolayers. Nat. Mater. 13(12), 1135-1142 (2014). https://doi. org/10.1038/Nmat4091

28. X.F. Li, M.W. Lin, J.H. Lin, B. Huang, A.A. Puretzky et al., Two-dimensional GaSe/MoSe ${ }_{2}$ misfit bilayer heterojunctions by van der Waals epitaxy. Sci. Adv. 2(4), 1501882 (2016). https://doi.org/10.1126/sciadv.1501882

29. L. Fu, Y.Y. Sun, N. Wu, R.G. Mendes, L.F. Chen et al., Direct growth of $\mathrm{MoS}_{2} / \mathrm{h}-\mathrm{BN}$ heterostructures via a sulfide-resistant 
alloy. ACS Nano 10(2), 2063-2070 (2016). https://doi. org/10.1021/acsnano.5b06254

30. B.Y. Zheng, C. Ma, D. Li, J.Y. Lan, Z. Zhang et al., Band alignment engineering in two-dimensional lateral heterostructures. J. Am. Chem. Soc. 140(36), 11193-11197 (2018). https ://doi.org/10.1021/jacs.8b07401

31. P.K. Sahoo, S. Memaran, Y. Xin, L. Balicas, H.R. Gutierrez, One-pot growth of two-dimensional lateral heterostructures via sequential edge-epitaxy. Nature 553(7686), 63-67 (2018). https://doi.org/10.1038/nature25155

32. Z.W. Zhang, P. Chen, X.D. Duan, K.T. Zang, J. Luo, X.F. Duan, Robust epitaxial growth of two-dimensional heterostructures, multiheterostructures, and superlattices. Science 357(6353), 788-792 (2017). https://doi.org/10.1126/scien ce.aan 6814

33. C. Gong, H.J. Zhang, W. Wang, L. Colombo, R.M. Wallace, K. Cho, Band alignment of two-dimensional transition metal dichalcogenides: application in tunnel field effect transistors. Appl. Phys. Lett. 107(13), 053513 (2015). https ://doi.org/10.1063/1.4932088

34. F. Withers, O. Del Pozo-Zamudio, A. Mishchenko, A.P. Rooney, A. Gholinia et al., Light-emitting diodes by bandstructure engineering in van der Waals heterostructures. Nat. Mater. 14(3), 301-306 (2015). https://doi.org/10.1038/ nmat4205

35. W.G. Xu, W.W. Liu, J.F. Schmidt, W.J. Zhao, X. Lu et al., Correlated fluorescence blinking in two-dimensional semiconductor heterostructures. Nature 541(7635), 62-67 (2017). https://doi.org/10.1038/nature20601

36. Y. Hattori, T. Taniguchi, K. Watanabe, K. Nagashio, Determination of carrier polarity in Fowler-Nordheim tunneling and evidence of fermi level pinning at the hexagonal boron nitride/metal interface. ACS Appl. Mater. Interfaces 10(14), 11732-11738 (2018). https://doi.org/10.1021/acsami.7b184 54

37. P. Bampoulis, R. van Bremen, Q.R. Yao, B. Poelsema, H.J.W. Zandvliet, K. Sotthewes, Defect dominated charge transport and fermi level pinning in $\mathrm{MoS}_{2} /$ metal contacts. ACS Appl. Mater. Interfaces 9(22), 19278-19286 (2017). https://doi.org/10.1021/acsami.7b02739

38. T. Le Quang, V. Cherkez, K. Nogajewski, M. Potemski, M.T. Dau, M. Jamet, P. Mallet, J.Y. Veuillen, Scanning tunneling spectroscopy of van der Waals graphene/semiconductor interfaces: absence of Fermi level pinning. 2D Mater. 4(3), 035019 (2017). https://doi.org/10.1088/20531583/aa7b03

39. C.R. Woods, L. Britnell, A. Eckmann, R.S. Ma, J.C. Lu, Commensurate-incommensurate transition in graphene on hexagonal boron nitride. Nat. Phys. 10(6), 451-456 (2014). https://doi.org/10.1038/Nphys2954

40. B. Li, L. Huang, M.Z. Zhong, Y. Li, Y. Wang, J.B. Li, Z.M. Wei, Direct vapor phase growth and optoelectronic application of large band offset $\mathrm{SnS}_{2} / \mathrm{MoS}_{2}$ vertical bilayer heterostructures with high lattice mismatch. Adv. Electron. Mater. 2(11), 1600298 (2016). https://doi.org/10.1002/aelm.20160 0298
41. K.H. Liu, L.M. Zhang, T. Cao, C.H. Jin, D.A. Qiu et al., Evolution of interlayer coupling in twisted molybdenum disulfide bilayers. Nat. Commun. 5, 4966 (2014). https://doi. org/10.1038/ncomms5966

42. H.J. Tan, W.S. Xu, Y.W. Sheng, C.S. Lau, Y. Fan et al., Lateral graphene-contacted vertically stacked $\mathrm{WS}_{2} / \mathrm{MoS}_{2}$ hybrid photodetectors with large gain. Adv. Mater. 29(46), 1702917 (2017). https://doi.org/10.1002/adma.201702917

43. F. Gong, W.J. Luo, J.L. Wang, P. Wang, H.H. Fang et al., High-sensitivity floating-gate phototransistors based on $\mathrm{WS}_{2}$ and $\mathrm{MoS}_{2}$. Adv. Funct. Mater. 26(33), 6084-6090 (2016). https://doi.org/10.1002/adfm.201601346

44. A. Dankert, S.P. Dash, Electrical gate control of spin current in van der Waals heterostructures at room temperature. Nat. Commun. 8, 16093 (2017). https://doi.org/10.1038/ ncomms 16093

45. J.L. Wang, H.H. Fang, X.D. Wang, X.S. Chen, W. Lu, W.D. Hu, Recent progress on localized field enhanced two-dimensional material photodetectors from ultravioletvisible to infrared. Small 13(35), 1700894 (2017). https:// doi.org/10.1002/smll.201700894

46. M.Z. Bellus, F. Ceballos, H.Y. Chiu, H. Zhao, Tightly bound trions in transition metal dichalcogenide heterostructures. ACS Nano 9(6), 6459-6464 (2015). https://doi. org/10.1021/acsnano.5b02144

47. L.B. Drissi, F.Z. Ramadan, N.B.J. Kanga, Optoelectronic properties in $2 \mathrm{D} \mathrm{GeC}$ and $\mathrm{SiC}$ hybrids: DFT and many body effect calculations. Mater. Res. Express 5(1), 015061 (2018). https://doi.org/10.1088/2053-1591/aaa862

48. X.P. Hong, J. Kim, S.F. Shi, Y. Zhang, C.H. Jin et al., Ultrafast charge transfer in atomically thin $\mathrm{MoS}_{2} / \mathrm{WS}_{2}$ heterostructures. Nat. Nanotechnol. 9(9), 682-686 (2014). https ://doi.org/10.1038/Nnano.2014.167

49. H.H. Hung, J.S. Wu, K. Sun, C.K. Chiu, Engineering of many-body Majorana states in a topological insulator/swave superconductor heterostructure. Sci. Rep. 7, 3499 (2017). https://doi.org/10.1038/s41598-017-02493-7

50. P. Rivera, J.R. Schaibley, A.M. Jones, J.S. Ross, S.F. Wu et al., Observation of long-lived interlayer excitons in monolayer $\mathrm{MoSe}_{2}-\mathrm{WSe}_{2}$ heterostructures. Nat. Commun. 6, 6242 (2015). https://doi.org/10.1038/ncomms 7242

51. M. Li, D. Esseni, G. Snider, D. Jena, H.G. Xing, Single particle transport in two-dimensional heterojunction interlayer tunneling field effect transistor. J. Appl. Phys. 115(7), 074508 (2014). https://doi.org/10.1063/1.4866076

52. X. Yan, C.S. Liu, C. Li, W.Z. Bao, S.J. Ding, D.W. Zhang, P. Zhou, Tunable $\mathrm{SnSe}_{2} / \mathrm{WSe}_{2}$ heterostructure tunneling field effect transistor. Small 13(34), 1701478 (2017). https ://doi.org/10.1002/smll.201701478

53. T. Yamaoka, H.E. Lim, S. Koirala, X.F. Wang, K. Shinokita et al., Efficient photocarrier transfer and effective photoluminescence enhancement in type i monolayer $\mathrm{MoTe}_{2} /$ $\mathrm{WSe}_{2}$ heterostructure. Adv. Funct. Mater. 28(35), 1801021 (2018). https://doi.org/10.1002/adfm.201801021

54. Z. Cai, M. Cao, Z.P. Jin, K.Y. Yi, X.S. Chen, D.C. Wei, Large photoelectric-gating effect of two-dimensional 
van-der-Waals organic/tungsten diselenide heterointerface. NPJ 2D Mater. Appl. 2, 21 (2018). https://doi. org/10.1088/2053-1591/aaa862

55. S. Bertolazzi, D. Krasnozhon, A. Kis, Nonvolatile memory cells based on $\mathrm{MoS}_{2}$ /graphene heterostructures. ACS Nano 7(4), 3246-3252 (2013). https://doi.org/10.1021/nn305 9136

56. M.S. Choi, G.H. Lee, Y.J. Yu, D.Y. Lee, S.H. Lee, P. Kim, J. Hone, W.J. Yoo, Controlled charge trapping by molybdenum disulphide and graphene in ultrathin heterostructured memory devices. Nat. Commun. 4, 1624 (2013). https://doi. org/10.1038/ncomms2652

57. Y. Zhang, Y.B. Yuan, J.S. Huang, Detecting $100 \mathrm{fW} \mathrm{cm}^{-2}$ light with trapped electron gated organic phototransistors. Adv. Mater. 29(5), 1603969 (2017). https://doi. org/10.1002/adma.201603969

58. J.L. Wang, X.M. Zou, X.H. Xiao, L. Xu, C.L. Wang et al., Floating gate memory-based monolayer $\mathrm{MoS}_{2}$ transistor with metal nanocrystals embedded in the gate dielectrics. Small 11(2), 208-213 (2015). https://doi.org/10.1002/ smll.201401872

59. Y.P. Liu, W.S. Lew, Z.W. Liu, Observation of anomalous resistance behavior in bilayer graphene. Nanoscale Res. Lett. 12, 48 (2017). https://doi.org/10.1186/s1167 $1-016-1792-\mathrm{Z}$

60. Y.P. Liu, Z.W. Liu, W.S. Lew, Q.J. Wang, Temperature dependence of the electrical transport properties in fewlayer graphene interconnects. Nanoscale Res. Lett. 8, 335 (2013). https://doi.org/10.1186/1556-276X-8-335

61. Q.A. Vu, Y.S. Shin, Y.R. Kim, V.L. Nguyen, W.T. Kang et al., Two-terminal floating-gate memory with van der Waals heterostructures for ultrahigh on/off ratio. Nat. Commun. 7, 12725 (2016). https://doi.org/10.1038/ncomm s12725

62. H.L. Chen, X.W. Wen, J. Zhang, T.M. Wu, Y.J. Gong et al., Ultrafast formation of interlayer hot excitons in atomically thin $\mathrm{MoS}_{2} / \mathrm{WS}_{2}$ heterostructures. Nat. Commun. 7, 12512 (2016). https://doi.org/10.1038/ncomms 12512

63. X.W. Wen, H.L. Chen, T.M. Wu, Z.H. Yu et al., Ultrafast probes of electron-hole transitions between two atomic layers. Nat. Commun. 9, 1859 (2018). https://doi.org/10.1038/s4146 7-018-04291-9

64. A.T. Hanbicki, H.J. Chuang, M.R. Rosenberger, C.S. Hellberg, S.V. Sivaram, K.M. McCreary, I.I. Mazin, B.T. Jonker, Double indirect interlayer exciton in a $\mathrm{MoSe}_{2} / \mathrm{WSe}_{2}$ van der waals heterostructure. ACS Nano 12(5), 4719-4726 (2018). https://doi.org/10.1021/acsnano.8b01369

65. S. Latini, K.T. Winther, T. Olsen, K.S. Thygesen, Interlayer excitons and band alignment in $\mathrm{MoS}_{2} / \mathrm{hBN} / \mathrm{WSe} \mathrm{H}_{2}$ van der waals heterostructures. Nano Lett. 17(2), 938-945 (2017). https://doi.org/10.1021/acs.nanolett.6b04275

66. J.S. Ross, P. Rivera, J. Schaibley, E. Lee-Wong, H.Y. Yu et al., Interlayer exciton optoelectronics in a 2D heterostructure p-n junction. Nano Lett. 17(2), 638-643 (2017). https://doi. org/10.1021/acs.nanolett.6b03398
67. P. Rivera, K.L. Seyler, H.Y. Yu, J.R. Schaibley, J.Q. Yan et al., Valley-polarized exciton dynamics in a $2 \mathrm{D}$ semiconductor heterostructure. Science 351(6274), 688-691 (2016). https://doi.org/10.1126/science.aac7820

68. T. Deilmann, K.S. Thygesen, Interlayer trions in the $\mathrm{MoS}_{2} /$ $\mathrm{WS}_{2}$ van der Waals heterostructure. Nano Lett. 18(2), 14601465 (2018). https://doi.org/10.1021/acs.nanolett.7b05224

69. Y.P. Liu, W.S. Lew, L. Sun, Enhanced weak localization effect in few-layer graphene. Phys. Chem. Chem. Phys. 13(45), 20208-20214 (2011). https://doi.org/10.1039/c1cp2 2250c

70. L.Y. Ping, G. Sarjoosing, M. Chandrasekhar, L.W. Siang, W.S.J.A.N. Kai, Effect of magnetic field on the electronic transport in trilayer graphene. ACS Nano 4(12), 7087-7092 (2010). https://doi.org/10.1021/nn101296x

71. J.S. Qi, X. Li, Q. Niu, J. Feng, Giant and tunable valley degeneracy splitting in $\mathrm{MoTe}_{2}$. Phys. Rev. B 92(12), 121403 (2015). https://doi.org/10.1103/PhysRevB.92.121403

72. B.Z. Zhou, S.W. Ji, Z. Tian, W.J. Cheng, X.C. Wang, W.B. $\mathrm{Mi}$, Proximity effect induced spin filtering and gap opening in graphene by half-metallic monolayer $\mathrm{Cr}_{2} \mathrm{C}$ ferromagnet. Carbon 132, 25-31 (2018). https://doi.org/10.1016/j.carbo n.2018.02.044

73. P. Wei, S. Lee, F. Lemaitre, L. Pinel, D. Cutaia et al., Strong interfacial exchange field in the graphene/EuS heterostructure. Nat. Mater. 15(7), 711-716 (2016). https://doi. org/10.1038/Nmat4603

74. H.X. Yang, A. Hallal, D. Terrade, X. Waintal, S. Roche, M. Chshiev, Proximity effects induced in graphene by magnetic insulators: first-principles calculations on spin filtering and exchange-splitting gaps. Phys. Rev. Lett. 110(4), 046603 (2013). https://doi.org/10.1103/PhysRevLett.110.046603

75. K.L. Seyler, D. Zhong, B. Huang, X.Y. Linpeng, N.P. Wilson et al., Valley manipulation by optically tuning the magnetic proximity effect in $\mathrm{WSe}_{2} / \mathrm{CrI}_{3}$ heterostructures. Nano Lett. 18(6), 3823-3828 (2018). https://doi.org/10.1021/acs.nanol ett. $8 \mathrm{~b} 01105$

76. B. Huang, G. Clark, E. Navarro-Moratalla, D.R. Klein, R. Cheng, Layer-dependent ferromagnetism in a van der Waals crystal down to the monolayer limit. Nature 546(7657), 270273 (2017). https://doi.org/10.1038/nature22391

77. J.U. Lee, S. Lee, J.H. Ryoo, S. Kang, T.Y. Kim et al., Isingtype magnetic ordering in atomically thin $\mathrm{FePS}_{3}$. Nano Lett. 16(12), 7433-7438 (2016). https://doi.org/10.1021/acs.nanol ett. $6 \mathrm{~b} 03052$

78. J. Du, C.X. Xia, W.Q. Xiong, T.X. Wang, Y. Jia, J.B. Li, Two-dimensional transition-metal dichalcogenides-based ferromagnetic van der Waals heterostructures. Nanoscale 9(44), 17585-17592 (2017). https://doi.org/10.1039/c7nr06473j

79. Y.P. Liu, I. Yudhistira, M. Yang, E. Laksono, Y.Z. Luo et al., Phonon-mediated colossal magnetoresistance in graphene/ black phosphorus heterostructures. Nano Lett. 18(6), 33773383 (2018). https://doi.org/10.1021/acs.nanolett.8b00155

80. M. Bonilla, S. Kolekar, Y.J. Ma, H.C. Diaz, V. Kalappattil et al., Strong room-temperature ferromagnetism in $\mathrm{VSe}_{2}$ 
monolayers on van der Waals substrates. Nat. Nanotechnol. 13(4), 289-293 (2018). https://doi.org/10.1038/s4156 5-018-0063-9

81. Z.Y. Fei, B. Huang, P. Malinowski, W.B. Wang, T.C. Song et al., Two-dimensional itinerant ferromagnetism in atomically thin $\mathrm{Fe}_{3} \mathrm{GeTe}_{2}$. Nat. Mater. 17(9), 778-782 (2018). https ://doi.org/10.1038/s41563-018-0149-7

82. Y. Deng, Y. Yu, Y. Song, J. Zhang, N.Z. Wang et al., Gatetunable room-temperature ferromagnetism in two-dimensional $\mathrm{Fe}_{3} \mathrm{GeTe}_{2}$. Nature 563, 94-99 (2018). https://doi. org/10.1038/s41586-018-0626-9

83. C. Si, J. Zhou, Z.M. Sun, Half-metallic ferromagnetism and surface functionalization-induced metal-insulator transition in graphene-like two-dimensional $\mathrm{Cr}_{2} \mathrm{C}$ crystals. ACS Appl. Mater. Interfaces 7(31), 17510-17515 (2015). https://doi. org/10.1021/acsami.5b05401

84. M. Kan, J. Zhou, Q. Sun, Y. Kawazoe, P. Jena, The intrinsic ferromagnetism in a $\mathrm{MnO}_{2}$ monolayer. J. Phys. Chem. Lett. 4(20), 3382-3386 (2013). https://doi.org/10.1021/jz401 7848

85. Y. Zhang, J.M. Pang, M.G. Zhang, X. Gu, L. Huang, Twodimensional $\mathrm{Co}_{2} \mathrm{~S}_{2}$ monolayer with robust ferromagnetism. Sci. Rep. 7, 7 (2017). https://doi.org/10.1038/s41598-01700035-9

86. M. Ormaza, L. Fernandez, M. Ilyn, A. Magana, B. Xu et al., High temperature ferromagnetism in a $\mathrm{GdAg}_{2}$ monolayer. Nano Lett. 16(7), 4230-4235 (2016). https://doi. org/10.1021/acs.nanolett.6b01197

87. M. Kan, S. Adhikari, Q. Sun, Ferromagnetism in $\mathrm{MnX}_{2}$ $(\mathrm{X}=\mathrm{S}, \mathrm{Se}$ ) monolayers. Phys. Chem. Chem. Phys. 16(10), 4990-4994 (2014). https://doi.org/10.1039/c3cp55146f

88. Y.P. Liu, W.S. Lew, S. Goolaup, H.F. Liew, S.K. Wong, T.J. Zhou, Observation of oscillatory resistance behavior in coupled Bernal and rhombohedral stacking graphene. ACS Nano 5(7), 5490-5498 (2011). https://doi.org/10.1021/nn200771e

89. S. Roche, J. Åkerman, B. Beschoten, J.C. Charlier, M. Chshiev et al., Graphene spintronics: the European Flagship perspective. 2D Mater. 2(3), 030202 (2015). https:// doi.org/10.1088/2053-1583/2/3/030202

90. M.W. Si, P.Y. Liao, G. Qiu, Y.Q. Duan, P.D.D. Ye, Ferroelectric field-effect transistors based on $\mathrm{MoS}_{2}$ and $\mathrm{CuInP}_{2} \mathrm{~S}_{6}$ two-dimensional van der Waals heterostructure. ACS Nano 12(7), 6700-6705 (2018). https://doi.org/10.1021/acsna no. 8 b01810

91. Y.P. Liu, K. Tom, X.W. Zhang, S. Lou, Y. Liu, J. Yao, Alloying effect on bright-dark exciton states in ternary monolayer $\mathrm{Mo}_{\mathrm{x}} \mathrm{W}_{1-\mathrm{x}} \mathrm{Se}_{2}$. New J. Phys. 19, 073018 (2017). https://doi. org/10.1088/1367-2630/aa6d39

92. Y.P. Liu, Q.L. Xia, J. He, Z.W. Liu, Direct observation of high photoresponsivity in pure graphene photodetectors. Nanoscale Res. Lett. 12, 93 (2017). https://doi.org/10.1186/ s11671-017-1827-0

93. Y.P. Liu, K. Tom, X. Wang, C.M. Huang, H.T. Yuan et al., Dynamic control of optical response in layered metal chalcogenide nanoplates. Nano Lett. 16(1), 488-496 (2016). https ://doi.org/10.1021/acs.nanolett.5b04140
94. X.C. Yu, Z.G. Dong, Y.P. Liu, T. Liu, J. Tao et al., A high performance, visible to mid-infrared photodetector based on graphene nanoribbons passivated with $\mathrm{HfO}_{2}$. Nanoscale 8(1), 327-332 (2016). https://doi.org/10.1039/C5NR06869J

95. W.G. Luo, Y.F. Cao, P.G. Hu, K.M. Cai, Q. Feng et al., Gate tuning of high-performance InSe-based photodetectors using graphene electrodes. Adv. Opt. Mater. 3(10), 1418-1423 (2015). https://doi.org/10.1002/adom.201500190

96. Q. Lv, F. Yan, X. Wei, K. Wang, High-performance, selfdriven photodetector based on graphene sandwiched GaSe/ $\mathrm{WS}_{2}$ heterojunction. Adv. Opt. Mater. 6(2), 1700490 (2018). https://doi.org/10.1002/adom.201700490

97. M. Massicotte, P. Schmidt, F. Vialla, K.G. Schadler, A. Reserbat-Plantey et al., Picosecond photoresponse in van der Waals heterostructures. Nat. Nanotechnol. 11(1), 42-46 (2016)

98. X. Wang, L. Huang, Y. Peng, N. Huo, K. Wu et al., Enhanced rectification, transport property and photocurrent generation of multilayer $\mathrm{ReSe}_{2} / \mathrm{MoS}_{2} \mathrm{p}-\mathrm{n}$ heterojunctions. Nano Res. 9(2), 507-516 (2016). https://doi.org/10.1007/s1227 4-015-0932-6

99. X. Wei, F.G. Yan, Q.S. Lv, C. Shen, K.Y. Wang, Fast gatetunable photodetection in the graphene sandwiched $\mathrm{WSe}_{2} /$ GaSe heterojunctions. Nanoscale 9(24), 8388-8392 (2017). https://doi.org/10.1039/C7NR03124F

100. F.G. Yan, L.X. Zhao, A.L. Patane, P.A. Hu, X. Wei et al., Fast, multicolor photodetection with graphene-contacted p-GaSe/nInSe van der Waals heterostructures. Nanotechnology 28, 27 (2017). https://doi.org/10.1088/1361-6528/aa749e

101. K. Zhang, X. Fang, Y.L. Wang, Y. Wan, Q.J. Song et al., Ultrasensitive near-infrared photodetectors based on a graphene- $\mathrm{MoTe}_{2}$-graphene vertical van der Waals heterostructure. ACS Appl. Mater. Interfaces 9(6), 5392-5398 (2017). https://doi.org/10.1021/acsami.6b14483

102. X. Wei, F.G. Yan, C. Shen, Q.S. Lv, K.Y. Wang, Photodetectors based on junctions of two-dimensional transition metal dichalcogenides. Chin. Phys. B. 26(3), 038504 (2017). https ://doi.org/10.1088/1674-1056/26/3/038504

103. C.H. Lee, G.H. Lee, A.M. van der Zande, W.C. Chen, Y.L. Li et al., Atomically thin p-n junctions with van der Waals heterointerfaces. Nat. Nanotechnol. 9(9), 676-681 (2014). https://doi.org/10.1038/nnano.2014.150

104. W.H. Wu, Q. Zhang, X. Zhou, L. Li, J.W. Su, F.K. Wang, T.Y. Zhai, Self-powered photovoltaic photodetector established on lateral monolayer $\mathrm{MoS}_{2}-\mathrm{WS}_{2}$ heterostructures. Nano Energy 51, 45-53 (2018). https://doi.org/10.1016/j. nanoen.2018.06.049

105. C. Choi, M.K. Choi, S.Y. Liu, M.S. Kim, O.K. Park et al., Human eye-inspired soft optoelectronic device using highdensity $\mathrm{MoS}_{2}-$ graphene curved image sensor array. Nat. Commun. 8, 1664 (2017). https://doi.org/10.1038/s4146 7-017-01824-6

106. F. Wang, Z.X. Wang, K. Xu, F.M. Wang, Q.S. Wang, Y. Huang, L. Yin, J. He, Tunable GaTe-MoS 2 van der Waals $\mathrm{p}-\mathrm{n}$ junctions with novel optoelectronic performance. Nano Lett. 15(11), 7558-7566 (2015). https://doi.org/10.1021/acs. nanolett.5b03291 
107. M.S. Long, E.F. Liu, P. Wang, A.Y. Gao, H. Xia et al., Broadband photovoltaic detectors based on an atomically thin heterostructure. Nano Lett. 16(4), 2254-2259 (2016). https://doi. org/10.1021/acs.nanolett.5b04538

108. K. Roy, M. Padmanabhan, S. Goswami, T.P. Sai, G. Ramalingam, S. Raghavan, A. Ghosh, Graphene- $\mathrm{MoS}_{2}$ hybrid structures for multifunctional photoresponsive memory devices. Nat. Nanotechnol. 8(11), 826-830 (2013). https:// doi.org/10.1038/Nnano.2013.206

109. S.J. Zheng, E.X. Wu, Z.H. Feng, R. Zhang, Y. Xie et al., Acoustically enhanced photodetection by a black phosphorus$\mathrm{MoS}_{2}$ van der Waals heterojunction $\mathrm{p}-\mathrm{n}$ diode. Nanoscale 10(21), 10148-10153 (2018). https://doi.org/10.1039/c8nr0 $2022 \mathrm{a}$

110. Q.M. Wang, Z.Y. Yang, Graphene photodetector with polydiacetylenes acting as both transfer-supporting and lightabsorbing layers: flexible, broadband, ultrahigh photoresponsivity and detectivity. Carbon 138, 90-97 (2018). https://doi. org/10.1016/j.carbon.2018.05.054

111. Y.J. Liu, C. Liu, X.M. Wang, L. He, X.G. Wan et al., Photoresponsivity of an all-semimetal heterostructure based on graphene and $\mathrm{WTe}_{2}$. Sci. Rep. 8, 12840 (2018). https://doi. org/10.1038/s41598-018-29717-8

112. D. Xiang, T. Liu, J.L. Xu, J.Y. Tan, Z.H. Hu et al., Twodimensional multibit optoelectronic memory with broadband spectrum distinction. Nat. Commun. 9, 2966 (2018). https:// doi.org/10.1038/s41467-018-05397-w

113. D. Unuchek, A. Ciarrocchi, A. Avsar, K. Watanabe, T. Taniguchi, A. Kis, Room-temperature electrical control of exciton flux in a van der Waals heterostructure. Nature 560(7718), 340-344 (2018). https://doi.org/10.1038/s41586-018-0357-y

114. B. Dlubak, M.-B. Martin, R.S. Weatherup, H. Yang, C. Deranlot et al., Graphene-passivated nickel as an oxidation-resistant electrode for spintronics. ACS Nano 6(12), 10930-10934 (2012). https://doi.org/10.1021/nn304424x

115. M.Z. Iqbal, M.W. Iqbal, S. Siddique, M.F. Khan, S.M. Ramay, Room temperature spin valve effect in $\mathrm{NiFe} / \mathrm{WS}_{2} / \mathrm{Co}$ junctions. Sci. Rep. 6, 21038 (2016). https://doi.org/10.1038/ srep21038

116. Z. Wang, D. Sapkota, T. Taniguchi, K. Watanabe, D. Mandrus, A.F. Morpurgo, Tunneling spin valves based on $\mathrm{Fe}_{3} \mathrm{GeTe}_{2} / \mathrm{hBN} / \mathrm{Fe}_{3} \mathrm{GeTe}_{2}$ van der Waals heterostructures. Nano Lett. 18(7), 4303-4308 (2018). https://doi.org/10.1021/ acs.nanolett.8b01278

117. H. Zhang, M. Ye, Y. Wang, R. Quhe, Y. Pan et al., Magnetoresistance in $\mathrm{Co} / 2 \mathrm{D} \mathrm{MoS}_{2} / \mathrm{Co}$ and $\mathrm{Ni} / 2 \mathrm{D} \mathrm{MoS}_{2} / \mathrm{Ni}$ junctions. Phys. Chem. Chem. Phys. 18(24), 16367-16376 (2016). https ://doi.org/10.1039/C6CP01866A
118. V.M. Karpan, G. Giovannetti, P.A. Khomyakov, M. Talanana, A.A. Starikov et al., Graphite and graphene as perfect spin filters. Phys. Rev. Lett. 99(17), 176602 (2007). https://doi. org/10.1103/PhysRevLett.99.176602

119. M.Z. Iqbal, S. Siddique, G. Hussain, M.W. Iqbal, Room temperature spin valve effect in the $\mathrm{NiFe} / \mathrm{Gr}-\mathrm{hBN} / \mathrm{Co}$ magnetic tunnel junction. J. Mater. Chem. C 4(37), 8711-8715 (2016). https://doi.org/10.1039/C6TC03425J

120. D. Xiao, G.B. Liu, W.X. Feng, X.D. Xu, W. Yao, Coupled spin and valley physics in monolayers of $\mathrm{MoS}_{2}$ and other group-VI dichalcogenides. Phys. Rev. Lett. 108(19), 196802 (2012). https://doi.org/10.1103/PhysRevLett.108.196802

121. J. Lee, K.F. Mak, J. Shan, Electrical control of the valley Hall effect in bilayer $\mathrm{MoS}_{2}$ transistors. Nat. Nanotechnol. 11(5), 421-425 (2016). https://doi.org/10.1038/nnano.2015.337

122. K.F. Mak, K.L. McGill, J. Park, P.L. McEuen, The valley Hall effect in $\mathrm{MoS}_{2}$ transistors. Science 344(6191), 1489-1492 (2014). https://doi.org/10.1126/science.1250140

123. A.V. Stier, K.M. McCreary, B.T. Jonker, J. Kono, S.A. Crooker, Exciton diamagnetic shifts and valley Zeeman effects in monolayer $\mathrm{WS}_{2}$ and $\mathrm{MoS}_{2}$ to 65 Tesla. Nat. Commun. 7, 10643 (2016). https://doi.org/10.1038/ncomm s10643

124. L. Cai, J.F. He, Q.H. Liu, T. Yao, L. Chen et al., Vacancyinduced ferromagnetism of $\mathrm{MoS}_{2}$ Nanosheets. J. Am. Chem. Soc. 137(7), 2622-2627 (2015). https://doi.org/10.1021/ ja5120908

125. C. Zhao, T. Norden, P.Y. Zhang, P.Q. Zhao, Y.C. Cheng et al., Enhanced valley splitting in monolayer $\mathrm{WSe}_{2}$ due to magnetic exchange field. Nat. Nanotechnol. 12(8), 757-762 (2017). https://doi.org/10.1038/Nnano.2017.68

126. Y.K. Luo, J.S. Xu, T.C. Zhu, G.Z. Wu, E.J. McCormick et al., Opto-valleytronic spin injection in monolayer $\mathrm{MoS}_{2} / \mathrm{few}$-layer graphene hybrid spin valves. Nano Lett. 17(6), 3877-3883 (2017). https://doi.org/10.1021/acs.nanolett.7b01393

127. Y.P. Liu, H. Idzuchi, Y. Fukuma, O. Rousseau, Y. Otani, W.S. Lew, Spin injection properties in trilayer graphene lateral spin valves. Appl. Phys. Lett. 102(3), 033105 (2013). https://doi. org/10.1063/1.4776699

128. M.V. Kamalakar, C. Groenveld, A. Dankert, S.P. Dash, Long distance spin communication in chemical vapour deposited graphene. Nat. Commun. 6, 6766 (2015). https://doi. org/10.1038/ncomms7766

129. F.G. Yan, Z.M. Wei, X. Wei, Q.S. Lv, W.K. Zhu, K.Y. Wang, Toward high-performance photodetectors based on 2D materials: strategy on methods. Small Methods 2(5), 1700394 (2018). https://doi.org/10.1002/smtd.201700349 